\title{
Audiotactile interactions in temporal perception
}

\author{
Valeria Occelli - Charles Spence $\cdot$ Massimiliano Zampini
}

Published online: 12 March 2011

(C) Psychonomic Society, Inc. 2011

\begin{abstract}
In the present review, we focus on how commonalities in the ontogenetic development of the auditory and tactile sensory systems may inform the interplay between these signals in the temporal domain. In particular, we describe the results of behavioral studies that have investigated temporal resolution (in temporal order, synchrony/asynchrony, and simultaneity judgment tasks), as well as temporal numerosity perception, and similarities in the perception of frequency across touch and hearing. The evidence reviewed here highlights features of audiotactile temporal perception that are distinctive from those seen for other pairings of sensory modalities. For instance, audiotactile interactions are characterized in certain tasks (e.g., temporal numerosity judgments) by a more balanced reciprocal influence than are other modality pairings. Moreover, relative spatial position plays a different role in the temporal order and temporal recalibration processes for audiotactile stimulus pairings than for other modality pairings. The effect exerted by both the spatial arrangement of stimuli and attention on temporal order judgments is described. Moreover, a number of audiotactile interactions occurring during sensory-motor synchronization are highlighted. We also look at the audiotactile perception of rhythm and how it may be affected by musical training. The
\end{abstract}

\section{Occelli $(\bowtie)$}

Department of Cognitive Sciences and Education,

University of Trento,

Corso Bettini, 31,

38068 Rovereto, TN, Italy

e-mail: valeria.occelli@unitn.it

C. Spence

Oxford University,

Oxford, UK

\section{Zampini}

Center for Mind/Brain Sciences, Department of Cognitive

Sciences and Education, University of Trento,

Rovereto, TN, Italy differences emerging from this body of research highlight the need for more extensive investigation into audiotactile temporal interactions. We conclude with a brief overview of some of the key issues deserving of further research in this area.

Keywords Auditory - Tactile · Temporal · Frequency Audiotactile $\cdot$ Crossmodal similarities $\cdot$ Mechanoreception . Multisensory

\section{The boundaries between hearing and touch: the foundation of an analogy}

We continuously interact with environments that provide a large amount of multisensory information to our various senses. Researchers have now convincingly demonstrated that the inputs delivered by the different sensory channels tend to be bound together by the brain (see the section Research on hearing and touch: a multisensory perspective for a fuller discussion of this topic). Unlike the audiovisual and visuotactile sensory pairings, those interactions taking place at both the neuronal and behavioral level between audition and touch have, to date, been explored in far less detail (see Kitagawa \& Spence, 2006; Soto-Faraco \& Deco, 2009, for reviews of the extant literature). The paucity of research covering this modality pairing is rather surprising when one considers the wide range of everyday situations in which we experience - even though often in subtle and unconscious ways - the interplay between these two senses. Examples include perceiving the "auditory" buzzing and the itchy "tactile" sensation of an insect landing on the back of our neck; reaching for a mobile phone ringing and vibrating in our pocket. What is common to these situations is the exclusive - or, at the very least, predominant-reliance on cues provided by the 
nonvisual spatial senses. In addition to these anecdotal reports, there is a growing body of empirical evidence demonstrating the existence of important similarities between the senses of hearing and touch (e.g., Soto-Faraco \& Deco, 2009).

In his pioneering early work, von Békésy $(1955,1957$, 1959) drew a number of parallels between the senses of audition and touch, which turned out to be so close as to lead him to consider the sense of touch as constituting a reliable model for the study of functional features of audition. For instance, von Békésy (1955) noted that audition and vibrotaction are analogous with regard to the level of the encoding mechanisms at their respective receptor surfaces. Indeed, both the basilar membrane of the inner ear and the mechanoreceptors embedded in the skin respond to the same type of physical energy — namely, mechanical pressure having a specific vibratory rate (e.g., either touching the surface of the skin with a vibrating body or stimulating the stapes footplate of the ear determines the propagation of travelling waves; von Békésy, 1959; cf. Nicolson, 2005).

The analogies in the physiological mechanisms (see Corey, 2003; Gillespie \& Müller, 2009) underlying vibrotactile and auditory perception are likely rooted in the common origins of these two sensory systems (see Soto-Faraco \& Deco, 2009; and von Békésy, 1959, for reviews). From this particular point of view, many pieces of evidence might be informative with regard to the existence of favored links between hearing and touch, in both animals (e.g., Bleckmann, 2008; Peck, 1994; Popper, 2000) and humans (Marks, 1983; von Békésy, 1959). The onset of function within the systems involved in sensory processing occurs in the following order: from the somesthetic and vestibular modalities to the chemosensory (oral and nasal), the auditory, and lastly, the visual modalities (see Gottlieb, 1971; Lickliter, 2000; Lickliter \& Bahrick, 2000; see also Lagercrantz \& Changeux, 2009; see Fig. 1).

The order in which the modality-specific as well as multisensory neurons in the anterior ectosylvian sulcus emerge also follows a precise time course, from tactile responsive to auditory responsive, and finally, to visually responsive neurons (Wallace, Carriere, Perrault, Vaughan, \& Stein, 2006). Therefore, one cannot rule out the possibility that the line of development of the different sensory systems might not have some effect upon the successive strength, direction, and amount of reciprocal connections between them (e.g., Gregory, 1967; Katsuki, 1965; Lickliter \& Bahrick, 2000).

Although the assessment of the nature of the stimulation that takes place during prenatal life, as well as the responsivity of the fetus to such stimulation, is problematic, the human fetus has been shown to respond to simultaneous stimulation in different sensory modalities since very early in development (e.g., Kisilevsky, Muir, \& Low, 1992).
Interestingly, the responses of the human fetus - as measured by heart rate and body movements - show an increase when stimulation is both vibratory and auditory as compared with when stimulation occurs in just one sensory modality in isolation (cf. Kisilevsky \& Muir, 1991). Moreover, since the responsivity to vibroacoustic stimulation follows a specific maturational time line across gestation (Hoh, Park, Cha, \& Park, 2009), any perturbation of the pattern of responses elicited by this kind of stimulation is thought to have a relevant diagnostic function during complicated pregnancies (D’Elia, Pighetti, Vanacore, Fabbrocini, \& Arpaia, 2005; Morokuma et al., 2004). It is plausible that the presentation of stimuli in close temporal proximity could support the deployment during later development of the favored processing of specific co-occurring crossmodal sensory inputs (see Lecaunet \& Schaal, 1996). Moreover, since the human fetus can respond to vibrotactile and acoustic information by the third trimester (Kisilevsky, 1995), whereas visual information is not fully transduced prior to birth, it is likely that crossmodal temporal synchrony is primarily experienced for the pairing of somatosensory and auditory signals (Lewkowicz, 2000).

The evidence emerging from embryology research is related with the commonality of some physical properties which, according to von Békésy (1959), are shared between audition and touch, such as pitch, loudness, volume, roughness, distance, on-and-off effects, and rhythm. For instance, in one experiment, participants were presented with a pair of clicks (one to either ear, separated by a variable time interval). As the time difference was increased from zero, the sound seemed to travel from one side of the participant's head to the other (i.e., with a leftward or rightward direction), and the participants were asked to point to the direction from which the sound seemed to come. In certain conditions, air pulses were presented across the participant's forehead. By adjusting the magnitude and timing of either auditory clicks or spatially coincident air-puffs so that the skin sensations matched the sensations produced by the acoustic clicks as closely as possible, von Békésy (1959) succeeded in demonstrating that observers found it very difficult to phenomenologically discriminate between auditory and tactile stimuli when they appeared to come from the same direction. This result points to the existence of remarkable analogies - at least under certain specific conditions of stimulus presentationbetween the two senses, which could also possibly reflect how the signals from these two sensory modalities interact, at both the neural and behavioral levels.

In this review, a multisensory perspective will be adopted in order to provide an overview of those behavioral studies that have investigated interactions between auditory and tactile stimuli. The focus will be on the audiotactile interactions occurring within the temporal domain. Interest 


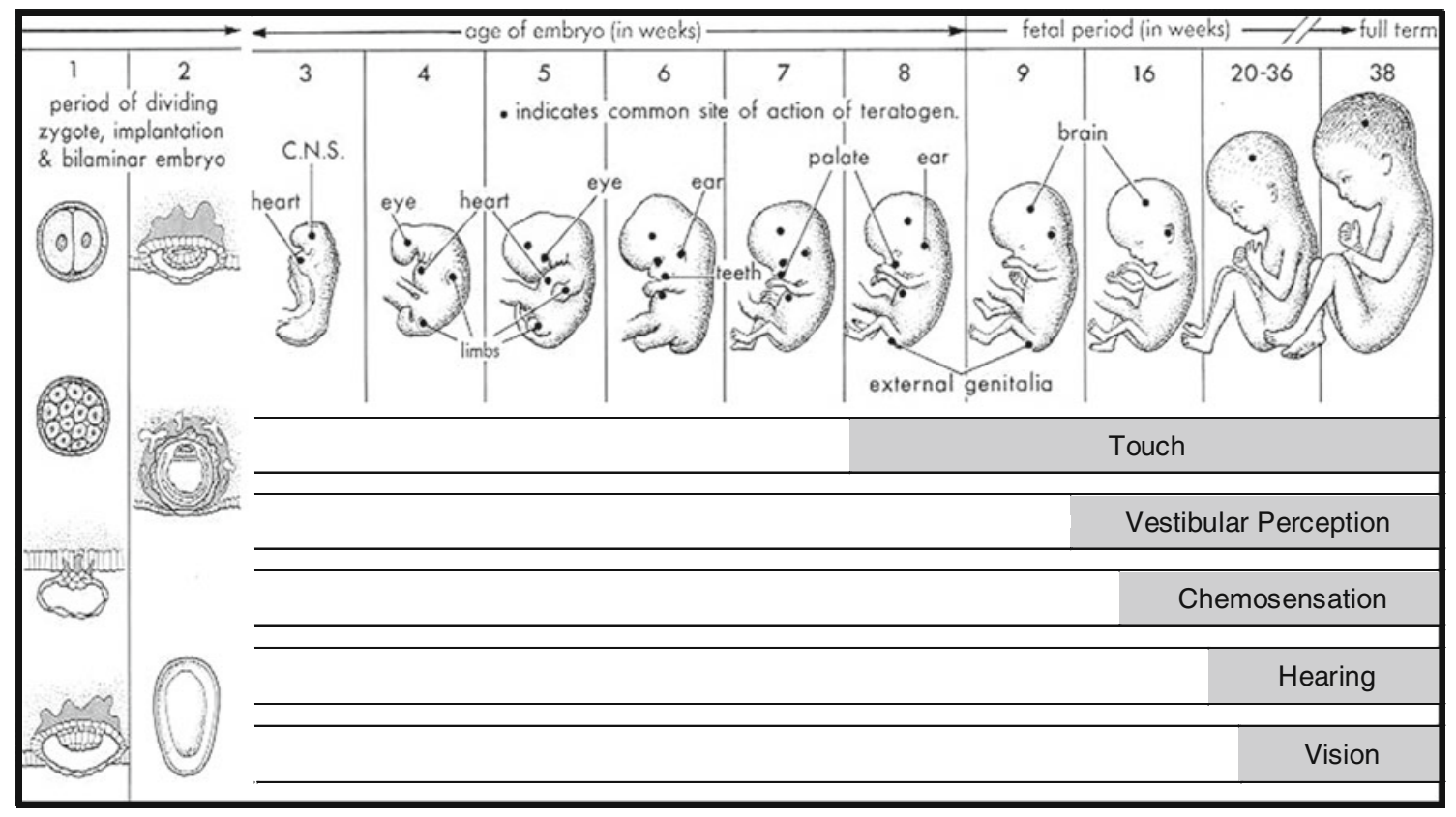

Fig. 1 The timeline of development of sensory systems during prenatal life (modified from Moore \& Persaud, 2008)

in temporal perception, both theoretical (e.g., Crystal, 2009; Droit-Volet \& Gil, 2009; Gibbon, 1977; Glicksohn, 2001; Pöppel, 1997; Wittmann, 2009) and experimental (e.g., Grondin, 2010; Mauk \& Buonomano, 2004), has been growing rapidly amongst the scientific community in recent years. According to certain authors, the emerging data are now consistent with humans having an amodal representation of time, one that is shared among different sensory modalities (cf. van Wassenhove, 2009). The remarks provided by van Wassenhove, though intriguing, are however currently confined to the auditory and visual modality pairing. In the present review, our aim has been to provide complementary coverage of the crossmodal nature of temporal perception by focusing on the audiotactile stimulus pairing instead.

\section{Research on hearing and touch: a multisensory perspective}

The process by which the human nervous system merges the available information into unique perceptual events is commonly known as multisensory integration (see Calvert, Spence, \& Stein, 2004, for a review). Operationally, multisensory integration has been defined at the neuronal level as "a statistically significant difference between the number of impulses evoked by a crossmodal combination of stimuli and the number evoked by the most effective of these stimuli individually" ("multisensory enhancement;" Stein \& Stanford, 2008, p. 255). This principle has been derived from a large body of studies conducted on the activity of neurons in the superior colliculus (SC), a midbrain structure involved in orienting behaviors. The specificity of this structure lies in the fact that it receives unisensory inputs from vision, touch, and audition (Rowland \& Stein, 2008; see Stein \& Meredith, 1993, for a review). On the basis of the available neurophysiological evidence, it is known that the processes by which the inputs delivered by different sensory pathways (e.g., visual, auditory, and somatosensory) are integrated are strongly affected by the spatial attributes of stimulation (this is known as the "spatial rule of multisensory integration;" Rowland \& Stein, 2008; Stein \& Stanford, 2008). Multisensory neurons have multiple excitatory receptive fields (RFs), one for each modality they are responsive to. Interestingly, the RFs of different sensory modalities overlap spatially (i.e., they are in approximate spatial register). Because of this characteristic, if multisensory inputs converge in this overlapping area, as when they originate from the same (or at least proximal) spatial locations, they can sometimes result in an enhancement of the neuronal response. If, on the other hand, the stimuli derive from spatially disparate locations, one of the stimuli may well fall within the inhibitory region of the neuron, thus determining a response depression. Moreover, multisensory enhancement is typically inversely related to the effectiveness of the single signals to be merged (this is known as the "law of inverse effectiveness;" Rowland \& Stein, 2008; Stein \& Stanford, 2008).

Particularly interesting in the present context, however, is the so-called "temporal rule of multisensory integration" (e.g., Calvert et al., 2004; Stein \& Meredith, 1993; Stein \& Stanford, 2008). Namely, only stimuli that occur in close temporal register (and hence that likely originate from the same event) result in response enhancement (i.e., evoke a 
rate of impulse firing that is significantly higher than the number of impulses evoked by the most effective of these stimuli when presented individually). Typically, the window of temporal tuning of multisensory neurons is a few tens to hundreds of milliseconds wide, with an optimal integration window estimated at approximately $250 \mathrm{~ms}$ (Meredith, Nemitz, \& Stein, 1987). By contrast, stimuli separated in time just induce responses that are comparable to those evoked by unisensory stimuli (e.g., Beauchamp, 2005; Meredith et al., 1987; see also Kayser \& Logothetis, 2007; van Wassenhove, 2009).

Along with the spatial rule and the law of inverse effectiveness, the temporal rule represents a core principle for the neural sensory integration processes and would possibly suggest a functional link between neuronal activity and the behavioral benefits of multisensory integration (see Holmes \& Spence, 2005; Stein \& Meredith, 1993; though see Holmes, 2007, 2009). Indeed, it has recently been demonstrated that multisensory integration yields a shortening of the latency between the stimulus arrival and the response elicited in an SC neuron (Rowland, Quessy, Stanford, \& Stein, 2007). This very early effect parallels the so-called "initial response enhancement," according to which the response enhancement is largest at the beginning of the response (Rowland $\&$ Stein, 2008). Both of these neuronal effects trigger and speed up the process by which crossmodal sources of information are integrated as soon the inputs reach the SC. This process results in faster behavioral responses to multisensory events as compared with those evoked by unisensory events (Rowland \& Stein, 2008). The links between the neuronal activity and its behavioral effects that have emerged in temporal perception tasks will be discussed more extensively in the sections that follow.

\section{Temporal resolution and temporal order}

To the best of our knowledge, one of the first attempts to assess temporal perception within hearing and touch dates back to the 1960s, when Gescheider (1966, 1967a, 1970) measured auditory and tactile temporal resolution (see also von Békésy, 1959). In a series of studies, Gescheider (1966, 1967a, 1970) demonstrated that the skin and ear differed greatly in terms of their ability to resolve successive stimuli (i.e., the temporal resolution thresholds for pairs of brief stimuli presented in rapid succession were found to be $5-10$ times higher for cutaneous stimulation than for auditory stimulation). For instance, two stimuli of equal subjective intensity were perceived as being temporally discrete if they were separated by $\sim 2 \mathrm{~ms}$ for monaural and binaural stimulation, but by $\sim 10-12 \mathrm{~ms}$ for cutaneous stimulation (Gescheider, 1966, 1967a). Moreover, pairs of auditory stimuli separated by less than $30 \mathrm{~ms}$ were perceived as being more disparate in time than pairs of cutaneous stimuli separated by the same temporal interval (Gescheider, 1970). However, when intervals greater than $30 \mathrm{~ms}$ were used, pairs of events in both modalities were perceived as equally separated (Gescheider, 1967b).

While Gescheider (1967a, 1967b) compared the temporal perception of auditory and tactile stimuli by testing them separately, Hirsh and Sherrick (1961) conducted the very first study to compare people's ability to judge the temporal features of stimuli presented either within or across different pairs of sensory modalities. They used the temporal order judgment (TOJ) paradigm, in which participants are presented with pairs of stimuli at various different stimulus onset asynchronies (SOAs) and have to judge which stimulus appeared first. It should, however, be noted that the issue explored by Hirsh and Sherrick in their study differs slightly from the one investigated in Gescheider's studies (1967a, 1967b). Indeed, the investigation of the perception of simultaneity or of temporal order likely activates different neuronal mechanisms, whose involvement is traditionally measured through distinct psychophysical estimates (cf. Van Eijk, Kohlrausch, Juola, \& van de Par, 2010; van Wassenhove, 2009; Wackermann, 2007). The "fusion threshold" is defined as the frequency (expressed in $\mathrm{Hz}$ ) at which observers perceive multiple events to be steady, the "simultaneity threshold" is the time interval required for two events to be correctly perceived as successive or simultaneous in time, and the "temporal order threshold" is the amount of time required for two events to be correctly ordered in time.

By measuring the just noticeable differences (JNDs, traditionally defined as the smallest temporal interval at which people can accurately discriminate the temporal order of the stimuli on $75 \%$ of the trials), Hirsh and Sherrick (1961) surprisingly found that the temporal separation required to correctly judge the temporal order was approximately $20 \mathrm{~ms}$, in both unimodal (i.e., tactile, auditory, or visual; Experiments 1-3) and multisensory (e.g., audiotactile, audiovisual, and visuotactile; Experiment 4) conditions. Hirsh and Sherrick stated that:

"[W] hereas the time between successive stimuli that is necessary for the stimuli to be perceived as successive rather than simultaneous may depend upon the particular sense modality employed, the temporal separation that is required for the judgment of perceived temporal order is much longer and is independent of the sense modality employed." (p. 432)

As we will see, many subsequent studies have provided evidence that has turned out to be fundamentally inconsistent with these claims (see, e.g., Fujisaki \& Nishida, 2009; Spence, Baddeley, Zampini, James, \& Shore, 2003; 
Zampini et al., 2005; Zampini, Shore, \& Spence, 2003a, 2003b; see Table 1).

For instance, in one recent study, Fujisaki and Nishida (2009) addressed the question of whether people's temporal perception differs as a function of the stimulus modality pairing under investigation. In particular, the authors studied whether there is any difference in terms of the temporal resolution of audiotactile, audiovisual, and visuotactile combinations of stimuli made of either single pulses (whose frequency changed from 1.4 to $26.7 \mathrm{~Hz}$ ) or else repetitivepulse trains (whose frequency changed between 6.25 and $356.25 \mathrm{~Hz}$ ). In their experiment, they used a set of paradigms, traditionally used to assess temporal perception, such as a synchrony-asynchrony discrimination task, a simultaneity judgment task (SJ), and a TOJ task. In the synchronyasynchrony judgment task, participants were presented with stimulus pairs having only one of two magnitudes of asynchrony $-0 \mathrm{~ms}$ (synchrony) and $\mathrm{X}$ ms (asynchrony)within a single block. The participants had to discriminate between the two alternatives. They were provided with trialby-trial feedback regarding the accuracy of their responses. In the SJ task, pairs of crossmodal stimuli were presented, at a range of different stimulus onset asynchronies (SOAs), using the method of constant stimuli, and the participants had to judge whether the stimuli were presented simultaneously or successively. In the SJ task, the judgments are closely dependent on the criterion adopted by the participants (i.e., their subjective "simultaneous" category), whereas in the synchrony-asynchrony task, they might decide to adjust their judgments in light of the feedback received.

The results of the synchrony-asynchrony judgment and SJ tasks, and-to a lesser extent-of the TOJ task, consistently showed that the temporal resolution of synchrony perception was significantly better for the audiotactile stimulus pairing than for either the audiovisual or visuotactile stimulus pairing. Interestingly, by applying their analysis of the data to those reported by Hirsh and Sherrick (1961), Fujisaki and Nishida (2009) found an analogous pattern of results, with the audiotactile stimulus pairing being processed with a higher degree of temporal resolution as compared with the other sensory pairings. Moreover, the fact that the superior temporal resolution reported for the audiotactile stimulus pairing in the TOJ task is smaller than in the other two tasks suggests a partial dissociation of the perception of temporal order from the perception of simultaneity (see Wackermann, 2007). Furthermore, the threshold required to discriminate synchrony from asynchrony is lower for single- than for repetitive-pulse

Table 1 Summary of the results of the temporal order judgment (TOJ) studies cited in the text

\begin{tabular}{|c|c|c|}
\hline Author(s) & Stimuli and Design & Results \\
\hline Hirsh and Sherrick (1961; Exp. 4) & $\begin{array}{l}\text { Three sessions, one for each stimulus pairing } \\
\text { (i.e., audiovisual, visuotactile, and audiotactile) }\end{array}$ & $\begin{array}{l}\text { JND of around } 20 \mathrm{~ms} \text { for all stimulus } \\
\text { combinations }\end{array}$ \\
\hline \multirow[t]{3}{*}{ Spence et al. (2003) } & $\begin{array}{l}\text { Exp. 1: Visuotactile pairs of stimuli, presented } \\
\text { either from the same or from different positions } \\
\text { in frontal space, uncrossed or crossed posture }\end{array}$ & $\begin{array}{l}\text { Exp 1: Uncrossed, JND: } 65 \mathrm{~ms} \text { (Same) vs. } \\
42 \mathrm{~ms} \text { (Different); } p<.0005\end{array}$ \\
\hline & \multirow{2}{*}{$\begin{array}{l}\text { Exp. 2: Audiovisual pairs of stimuli, presented } \\
\text { either from the same or from different positions } \\
\text { in frontal space }\end{array}$} & $\begin{array}{l}\text { Crossed, JND: } 50 \mathrm{~ms} \text { (Same) vs. } 58 \mathrm{~ms} \\
\text { (Different); n.s. }\end{array}$ \\
\hline & & $\begin{array}{l}\text { Exp 2: JND: } 53 \mathrm{~ms} \text { (Same) vs. } 42 \text { (Different); } \\
\quad p<.05\end{array}$ \\
\hline Kitagawa et al. (2005; Exp. 1) & $\begin{array}{l}\text { Audiotactile pairs of stimuli, presented either from } \\
\text { the same or from different positions in rear space }\end{array}$ & $\begin{array}{l}\text { JND: } 64 \mathrm{~ms} \text { (Same) vs. } 55 \mathrm{~ms} \text { (Different); } \\
\quad p<.05\end{array}$ \\
\hline \multirow[t]{3}{*}{ Zampini et al. (2005) } & \multirow{3}{*}{$\begin{array}{l}\text { Audiotactile pairs of stimuli, presented either } \\
\text { from the same or from different positions } \\
\text { in frontal space }\end{array}$} & $\begin{array}{l}\text { Exp 1: JND: } 75 \mathrm{~ms} \text { (Same) vs. } 82 \mathrm{~ms} \\
\text { (Different); } n . s .\end{array}$ \\
\hline & & $\begin{array}{l}\text { Exp 2: JND: } 45 \mathrm{~ms} \text { (Same) vs. } 42 \mathrm{~ms} \\
\text { (Different); } n . s .\end{array}$ \\
\hline & & $\begin{array}{l}\text { Exp 3: JND: } 44 \mathrm{~ms} \text { (Same) vs. } 44 \mathrm{~ms} \\
\text { (Different); n.s. }\end{array}$ \\
\hline \multirow[t]{3}{*}{ Occelli et al. (2008) } & \multirow{3}{*}{$\begin{array}{l}\text { Audiotactile pairs of stimuli, presented } \\
\text { either from the same or from different } \\
\text { positions in frontal space }\end{array}$} & Group X Relative Spatial Position, $p=.03$ \\
\hline & & $\begin{array}{l}\text { Sighted, JND: } 69 \mathrm{~ms} \text { (Same) vs. } 70 \mathrm{~ms} \\
\text { (Different); n.s. }\end{array}$ \\
\hline & & $\begin{array}{l}\text { Blind, JND: } 73 \mathrm{~ms} \text { (Same) vs. } 61 \mathrm{~ms} \\
\text { (Different); } p=.005\end{array}$ \\
\hline Fujisaki and Nishida (2009; Exp. 4) & $\begin{array}{l}\text { Four sessions (i.e., audiovisual, visuotactile, } \\
\text { audiotactile, tactile) }\end{array}$ & $\begin{array}{l}\text { JND: } 36 \text { ms (audiovisual), } 29 \mathrm{~ms} \text { (visuotactile), } \\
25 \mathrm{~ms} \text { (audiotactile), } 17 \mathrm{~ms} \text { (tactile); TT vs. } \\
\text { AV: } p=0.02 \text {; TT vs. VT: } p=0.02 \text {, no } \\
\text { other significant values }\end{array}$ \\
\hline
\end{tabular}


trains. This was found regardless of the stimulus combination used, possibly suggesting that both single-pulse and repetitive-pulse thresholds for the different stimulus combination may be coded by a common mechanism governing temporal resolution. If we assume that the threshold for single pulse trains indicates the width of the window of simultaneity reflecting the temporal precision of the temporal matching process, then the higher threshold observed for repetitivepulse trains could be suggestive of an increased risk of false matching by the participants in this condition (Fig. 2).

The fact that audiotactile processing has a higher temporal resolution than that of the other stimulus modality pairings can be ascribed, according to Fujisaki and Nishida (2009), to two different explanations, which are by no means necessarily mutually exclusive. The first explanation takes into account the difference in temporal resolution that exists between the various senses. Since vision is known to have a lower temporal resolution than either audition or touch (Welch \& Warren, 1980), whenever this sensory modality is involved, performance deteriorates. The alternative explanation takes into account the independent channels model proposed by Sternberg and Knoll (1973). According to their model, the perceived order of two stimuli is determined by evaluating the timing at which stimuli arrive at a central decision mechanism, or "comparator." Fujisaki and Nishida (2009) argue that the higher temporal resolution for audiotactile stimuli reflects the more rapid operation of this comparator for audiotactile pairs of signals. The supposed higher degree of similarity in the temporal profile of the auditory and tactile inputs could possibly induce a facilitation of the comparison of their temporal characteristics as compared with when matching between stimuli presented to other sensory modalities (see also Cappe, Morel, Barone, \& Rouiller, 2009; Hackett et al., 2007; Ley, Haggard, \& Yarrow, 2009; Wang, Lu, Bendor, \& Bartlett, 2008).

Moreover, Fujisaki and Nishida's (2009) study covers the issue of the relationship between synchrony discrimination and temporal order discrimination (cf. Fujisaki \&
Nishida, 2010). The link between these two kinds of perception is well described by Wackermann (2007):

Temporal experience is primarily experience of succession. The relations of temporal order between events, "A occurs before B," or "A occurs after B," constitute the most elementary form of temporal judgment, preceding a metrical concept of time scale. Our notion of time as a perfectly ordered universe of events implies that any two events, $\mathrm{A}$ and $\mathrm{B}$, are comparable as to their temporal order. If $\mathrm{A}$ occurs neither before $\mathrm{B}$ nor after $\mathrm{B}$, then the events $\mathrm{A}$ and $\mathrm{B}$ are simultaneous. (p. 22)

This definition suggests that the processing of synchrony and temporal order between sensory stimuli are highly related mechanisms. However, the minimum temporal interval necessary for two stimuli to be perceived as nonsimultaneous (defined as the "fusion threshold;" Exner, 1875) does not coincide with the time interval required to indicate their relative order (what is known as the "order threshold"). This fact has been taken to suggest the existence of multiple brain mechanisms for two different aspects of temporal discrimination (i.e., one for the integration of a unitary percept, another for the determination of succession between different percepts; Hirsh \& Fraisse, 1964; Piéron, 1952; van Wassenhove, 2009; see also the section Temporal synchrony and temporal recalibration).

The issue of a centralized versus distributed timing mechanism has been elegantly explored in a recent study by Fujisaki and Nishida (2010). The authors investigated whether the binding of synchronous attributes is specific to each attribute/sensory combination, or whether instead it occurs at a more central level. By using a psychophysical approach, they measured the processing speed of the judgments of the temporal relationship between two sequences of stimuli (i.e., cross-attribute phase judgments), either within single modalities or crossmodally. The rationale was that, in those cases in which the speed of binding

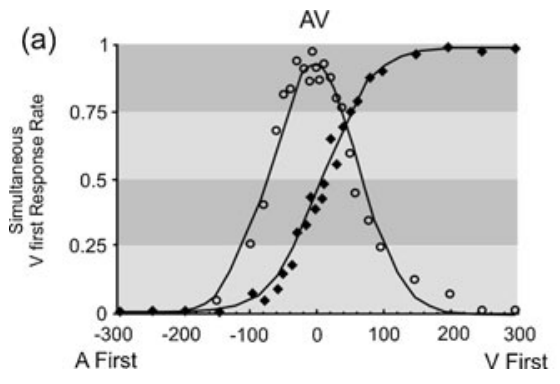

Fig. 2 Simultaneity judgments (SJs) and temporal order judgments (TOJs) for a audiovisual (AV), b visuotactile (VT), and c audiotactile (AT) stimulus pairs. Smooth curves represent the Gaussian function for the SJ data and the best cumulative Gaussian function for the TOJ data (modified from Fujisaki \& Nishida, 2009, Fig. 4). AT has a
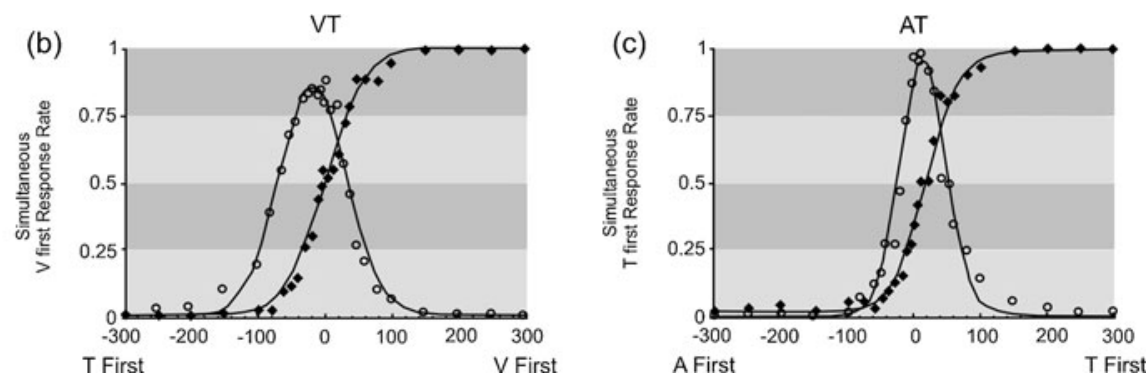

narrower range of simultaneous response for $\mathrm{SJ}$, and a steeper slope of psychometric function for TOJ as compared to both VT and AV stimulus pairings. Moreover, although both tasks are affected by stimulus combination, this effect is less pronounced in TOJ (vs. SJ) judgments (see Main Text for furher details) 
is high and varies as a function of different attribute combinations, the underlying mechanisms are likely to be peripheral and attribute specific. By contrast, a low and invariant binding speed is expected when considering a shared underlying mechanism. In Fujisaki and Nishida's (2010) study, participants had to perform a binding task and a synchrony-asynchrony discrimination task. In the first task, two sequences of stimuli were presented, each of which consisting of the repetitive alternation of two attributes (e.g., in the audiotactile condition, high- or low-pitched sounds were presented together with vibrations to the right or left index finger). The alternations always occurred synchronously between the two sequences, but the feature pairing varied as a function of the in-phase/reversedphase conditions. The participants had to judge which features were presented simultaneously (e.g., whether the pitch was high or low when the right finger was vibrated). In the synchronyasynchrony discrimination task, each stimulus sequence contained pulses at a given repetition rate, and the participants had to judge whether the pulses of the two sequences were presented synchronously or asynchronously. The results demonstrated that, whereas the temporal limit on cross-attribute binding was very low $(2-3 \mathrm{~Hz})$ and similar for all sensory modality combinations, the synchrony limit varied across the modality combinations (i.e., $4-5 \mathrm{~Hz}$ for audiovisual and visuotactile conditions, $7-9 \mathrm{~Hz}$ in the audiotactile condition).

Taken together, these results therefore suggest that crossmodal temporal binding and synchrony judgments are governed by different underlying neural mechanisms, with the first process being mediated by a central and amodal mechanism, whereas the perception of crossmodal synchrony appears to be mediated by a peripheral mechanism specific for each attribute combination (Weiss \& Scharlau, 2011). However, according to Fujisaki \& Nishida (2010), synchrony perception is also centrally represented, as demonstrated by the fact that the temporal limits of crossmodal synchrony perception are still much lower than the limits observed in the individual sensory modalities (cf. Fujisaki \& Nishida, 2005). Moreover, synchrony perception is only slightly affected by the attribute combination as long as the modality combination does not change. Therefore, the authors conjectured that both the capability to accurately extract salient changes in time in each sensory channel and to compare them across sensory modalities contributes to improving synchrony judgments. In the binding task, beyond the capability to process the "when" dimension, the capability to judge which combination of stimulus attributes are presented at the same time (the "what" dimension) is also important (see Renier et al., 2009; Yau, Olenczak, Dammann, \& Bensmaia, 2009). Thus, the crossmodal temporal binding and the synchrony judgment task would tap into different processes in the perception of an event, thus explaining the discrepancy in the performance observed in the two tasks. However, as pointed out by the authors themselves, the reason why the temporal limit should settle around $2-3 \mathrm{~Hz}$ in all of the conditions is unclear, and possibly involves a precise investigation of the timing of high-level sensory processing (Fujisaki \& Nishida, 2010).

\section{Spatial effects on the perception of temporal order}

An additional aspect to emerge from the literature on TOJ tasks is that audiotactile TOJs seem to be unaffected by the spatial disparity between the stimuli being judged. In a series of experiments, Zampini and his colleagues (Zampini et al., 2005) had participants perform a TOJ task on pairs of stimuli, one tactile and the other auditory, presented at varying SOAs. The stimuli could either be presented from either the same spatial location (i.e., both on the right or the left side of the participant's body midline) or different locations (i.e., one on the right and the other on the left side of the body midline). The results revealed that, contrary to what had been observed previously for audiovisual and visuotactile modality pairings (Spence et al., 2003; Zampini et al., 2003a, 2003b), the audiotactile version of the TOJ task was unaffected by whether the stimuli were presented from the same or different locations (sides). In previous studies, participants were found to be more sensitive (i.e., the results revealed smaller JNDs; Spence et al., 2003; Zampini et al., 2003a, 2003b) when the stimuli in the two modalities were presented from different spatial positions rather than from the same position (Fig. 3).

The null effect of relative spatial position reported by Zampini et al. (2005) suggests that the audiotactile stimulus pairing may be somehow "less spatial" than the other multisensory pairings involving vision as one of the sensory modalities. These data add to previous research documenting a reduced magnitude of spatial interaction effects for this particular pair of modalities, as compared with the audiovisual and visuotactile pairings, possibly suggesting a finer spatial resolution of visual stimuli than of the auditory and tactile systems (e.g., Eimer, 2004; Gondan, Niederhaus, Rösler, \& Röder, 2005; Lloyd, Merat, McGlone, \& Spence, 2003; Murray et al., 2005).

Subsequent studies have, however, partially undermined this conclusion, demonstrating instead that the spatial arrangement of the stimuli, and the portion of space stimulated, can differentially affect the nature of the audiotactile interactions that may be observed (Kitagawa, Zampini, \& Spence, 2005; Occelli, Spence, \& Zampini, 2008). In Kitagawa et al.'s (Experiment 1) audiotactile TOJ experiment, the participants had to judge the temporal order of pairs of auditory and tactile stimuli presented from the 


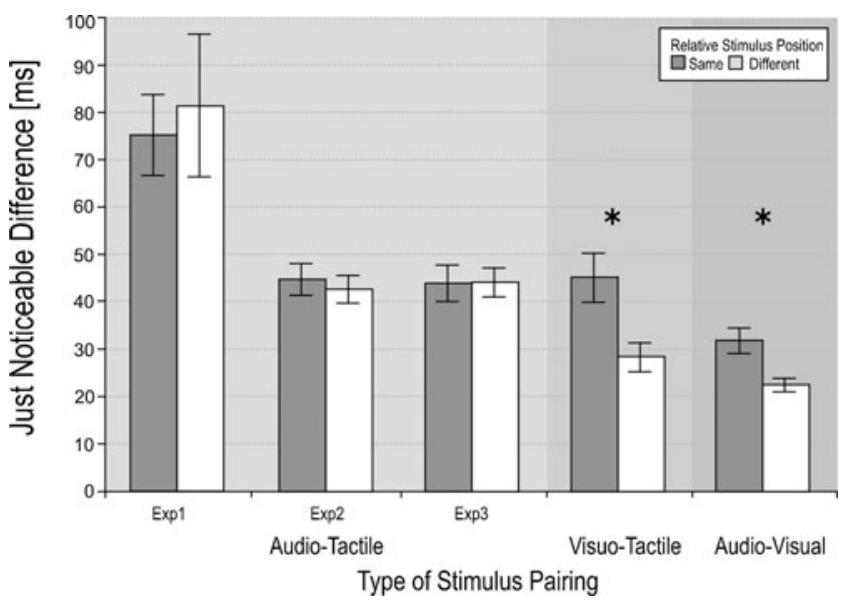

Fig. 3 Just noticeable differences (JNDs) for the audiotactile stimulus pairs presented in Experiments 1-3 of Zampini et al.'s (2005) study compared with pairs of visuotactile stimuli (Spence et al., 2003, Experiment 1), and with audiovisual stimulus pairs (Zampini et al., 2003a, Experiment 1) in crossmodal TOJ studies. The error bars represent the within-observer standard errors of the means. The presentation of audiotactile stimulus pairs from different positions did not facilitate participants' performance. By contrast, when visuotactile or audiovisual stimuli were presented from different positions (e.g., sides), performance was significantly better (i.e., the JND was smaller) than when the stimuli were presented from the same position (indicated by asterisks; modified from Zampini et al., 2005, Fig. 2)

left and/or right of fixation at varying SOAs and report which modality had been presented first on each trial. The auditory stimuli were presented from loudspeaker cones, whereas the tactile stimuli were delivered via electrotactile stimulators attached to the participants' earlobes. The results highlighted higher sensitivity (i.e., a lower JND), for stimuli presented from different sides rather than from the same side (i.e., 55 vs. $64 \mathrm{~ms}$ ).

The discrepancy between the results reported in audiotactile TOJ tasks for stimuli presented from the back and from frontal space (Kitagawa et al., 2005; Zampini et al., 2005) can be explained by taking into account the crucial role of vision in the processing of spatial information in frontal space (Eimer, 2004). That is, audiotactile interactions may be somewhat less "spatial" than other multisensory interactions involving vision as one of the component sensory modalities (e.g., think of audiovisual and visuotactile stimulus pairings; see Spence et al., 2003; Zampini et al., 2003a, 2003b), or when audiotactile stimuli are presented at locations in which visual cues are normally available. By contrast, the lack of visual cues, just as in Kitagawa et al.'s study, may have contributed to a better coding of auditory and tactile spatial cues, which, in turn, could have induced benefits in the processing of their temporal features.

This explanation has recently received further support from another study by Occelli et al. (2008). There, the potential modulatory effect of relative spatial position on audiotactile TOJs was examined as a function of the visual experience of the participants using the paradigm developed by Zampini et al. (2005). The results of Occelli et al.'s study demonstrated that although the performance of the sighted (blindfolded) participants was unaffected by whether or not the two stimuli were presented from the same spatial location, thus replicating Zampini et al.'s earlier findings, the blind participants (regardless of the age of onset of their blindness) were significantly more accurate when the auditory and tactile stimuli were presented from different spatial positions rather than from the same position (see Table 1). Thus, the relative spatial position from which the stimuli were presented had a selective effect on the performance of the blind, but not on the performance of the sighted participants. This pattern of results suggests that the exclusive reliance on those sensory modalities that are typically considered less adequate for conveying spatial information (see Welch \& Warren, 1980) failed to induce any advantage in terms of the performance of the blindfolded sighted participants. On the contrary, visual deprivation results in an enhancement of the ability to use the spatial cues available in the intact residual senses (e.g., hearing and touch; see also Collignon, Renier, Bruyer, Tranduy, \& Veraart, 2006; Röder, Rösler, \& Spence, 2004; Röder et al., 1999). Taken together, these data therefore reveal that the absence of vision (Occelli et al., 2008) or of visual information, as for stimulation occurring behind a participant's head (Farnè \& Làdavas, 2002; Kitagawa et al., 2005), seems to be related to more prevalent audiotactile spatial interactions than those occurring in frontal space (Zampini et al., 2005). Moreover, the processing of the spatial cues within touch and audition is improved by presenting the stimuli from that portion of space in which visual cues are typically unavailable (Kitagawa et al., 2005; Experiment 1) or absent as a result of blindness (e.g., Collignon et al., 2006; Occelli et al., 2008; Röder et al., 2004; Röder \& Rösler, 2004).

\section{Temporal synchrony and temporal recalibration}

As was already highlighted (see the section Research on Hearing and Touch: A Multisensory Perspective), multisensory integration can take place between stimuli that are not temporally coincident, but which fall within the "temporal window" of integration (Meredith et al., 1987; see also Spence, in press), thus indicating that the merging of information from different modalities can overcome the differences of the senses in terms of conduction speeds, response latencies, and neural processing times (e.g., Lestienne, 2001; Nicolas, 1997; Vroomen \& Keetels, 2010). Even though its extent is still a matter of some 
debate (e.g., Vatakis \& Spence, 2010; Vroomen \& Keetels, 2010), the existence of a window of temporal tolerance has not only empirical but also theoretical implications (e.g., Pöppel, 2009; van Wassenhove, 2009). Indeed, it implies that the concept of "temporal coincidence" (or "time point;" von Baer, 1864) in perception cannot be experienced in real life, but is rather a construct coinciding not with a specific point in time, but with a window of time. It follows from this that two stimuli falling within this temporal window are likely to be bound together into a single multisensory percept (see also Pöppel, Schill, \& von Steinbüchel, 1990). Next, those studies that have investigated the temporal window of integration between auditory and tactile signals will be reviewed.

One series of experiments has investigated people's perceptual sensitivity to simultaneity between haptic and auditory events and whether this would be significantly affected by the physical characteristics of the stimuli that were presented. To address this question, realistic stimulation conditions - such as a hammer hitting a surface or a drum being tapped-were followed by their auditory consequences, and were either executed (Adelstein, Begault, Anderson, \& Wenzel, 2003) or filmed (Levitin, MacLean, Mathews, \& Chu, 1999). Despite the high between-participants performance variability (see also Begault, Adelstein, McClain, \& Anderson, 2005), in both cases, the performance metrics calculated on basis of SJ data observed were significantly different from zero. It can be noted, however, that the values reported in these studies are much smaller than the $80 \mathrm{~ms}$ reported in Zampini et al.'s (2005) audiotactile TOJ study. Thus, even though the optimal impression of simultaneity for auditory and tactile stimuli is not perceived when the two stimuli are presented synchronously, it would seem that the conditions of stimulation have an effect in modulating the perceived relative temporal relationship between the stimuli, as measured by JNDs in synchrony/asynchrony tasks (see also Fink, Ulbrich, Churan, \& Wittmann, 2006; Vroomen \& Keetels, 2010, for other factors affecting the perception of intersensory synchrony). In particular, the use of ecological stimuli, such as those used in the studies of Adelstein et al. and Levitin et al., raises the question of how causality influences multisensory integration. On the basis of a number of recent audiovisual studies, it is known that when the stimulus presented in one modality in some sense predicts the stimulus in the other, multisensory integration is often enhanced (see Mitterer \& Jesse, 2010; Schutz \& Kubovy, 2009; Vroomen \& Stekelenberg, 2010). A similar effect may have affected audiotactile integration in the previously described studies (Adelstein et al., 2003; Levitin et al., 1999), with possibly boosted consequences (i.e., higher tendency to merge multisensory inputs) where visual cues were involved, as in Levitin et al.'s study.
Closely related to the studies just described are those that have assessed the mechanisms of temporal recalibration/ adaption between auditory and tactile stimuli (Hanson, Heron, \& Whitaker, 2008; Harrar \& Harris, 2008; Levitin et al., 1999; Navarra, Soto-Faraco, \& Spence, 2007; Virsu, Oksanen-Hennah, Vedenpää, Jaatinen, \& Lahti-Nuuttila, 2008). It has been observed that inputs from different sensory modalities that refer to the same external event (or occur at the same time) will likely reach the cortex at different times, due to differences in the speed of transmission of the signals through different sensory systems (King, 2005; Macefield, Gandevia, \& Burke, 1989; Schroeder \& Foxe, 2004, 2005; Spence, Shore, \& Klein, 2001; Spence \& Squire, 2003). It follows from this observation that our perceptual systems need to be able to accommodate a certain degree of asynchrony between the information arriving through different channels.

In the literature on crossmodal integration, it has been demonstrated that the point of subjective simultaneity (PSS; "amount of time by which one stimulus has to precede or follow the other order for the two stimuli to be perceived as simultaneous;" Spence \& Parise, 2010, p. 365) measure can be significantly affected by adaptation to asynchrony (see Vroomen \& Keetels, 2010, for a review). This process is typically assessed by measuring participants' perceptions of crossmodal simultaneity both before and after exposure to a constant temporal discrepancy between the stimuli that happen to be presented in the two modalities. During the exposure phase of such studies, the perception of asynchronous stimuli should be progressively realigned. As a consequence, the perception of simultaneity changes, in a way that, after exposure, the impression of asynchrony is reduced. There are two candidate mechanisms for the process of temporal recalibration: (a) The realignment of sensory neural signals in time, with the processing of one of the sensory modalities shifting in time toward the other; and (b) The widening of the temporal window for multisensory integration (see Vroomen \& Keetels, 2010).

In their study, Navarra et al. (2007) investigated whether exposure to audiotactile asynchrony would induce temporal recalibration between the processing of auditory and tactile stimuli. The participants in their study had to perform an audiotactile TOJ task both before and after an exposure phase in which paired auditory and vibrotactile stimuli could be presented either simultaneously or with the sound leading the vibration by $75 \mathrm{~ms}$. In the exposure phase of the experiment, in order to ensure that participants attended to both auditory and tactile stimuli, they had to perform a control task involving the detection of stimuli that were longer than the standards. Navarra et al.'s results highlighted the fact that exposure to audiotactile asynchrony induced a temporal adaptation aftereffect that influenced the temporal processing of the subsequently presented auditory and tactile 
stimuli. More precisely, the minimal interval necessary to correctly judge the temporal order of the stimuli was larger after exposure to the desynchronized trains of stimuli (JND = $48 \mathrm{~ms}$ ) than after exposure to the synchronous stimulus trains $(\mathrm{JND}=36 \mathrm{~ms})$, whereas no differences were observed in the PSS (i.e., 11 vs. $5 \mathrm{~ms}$ ). This result differs from the temporal adaptation process taking place between visual and auditory stimuli, as assessed using similar experimental methods (e.g., Fujisaki, Shimojo, Kashino, \& Nishida, 2004; Vroomen, Keetels, de Gelder, \& Bertelson, 2004). It thus seems that the audiotactile temporal window is flexible and can be widened in order to compensate for the asynchronies occurring between these stimuli, differently from the audiovisual condition, in which a temporal realignment process seems to take place (cf. Fujisaki et al., 2004). Navarra et al. suggested that the discrepancy between the results could be explained by considering the rare occurrence and the small magnitude of the asynchronies occurring between hearing and touch experienced in everyday life. According to this speculation, the widening of the temporal window of multisensory integration could be considered as a nonspecific mechanism that allows for the integration of -infrequentlyexperienced - audiotactile stimuli presented in close temporal proximity, and the temporal realignment as a more specific compensatory mechanism, suitable for coping with the relatively large asynchronies that may occur between visual and auditory stimuli (Navarra et al., 2007).

Contrasting results have, however, been reported recently. Harrar and Harris (2008) compared the changes in the perception of simultaneity for three different combinations of stimulus modality (i.e., audiotactile, audiovisual, and visuotactile) as a function of the exposure to asynchronous stimulus pairs, which were presented in each of the three stimulus combinations. In contrast with Navarra et al.'s (2007) results, no temporal adaptation (i.e., neither a change of the JND nor of the PSS) was observed for the audiotactile pairings following exposure to any of the three stimulus combinations. According to Harrar and Harris, this discrepancy could be attributed to methodological differences. Specifically, in their study, the tactile (not the auditory, as in Navarra et al.'s [2007] study) stimulus led within the asynchronous pairs. Moreover, the exposure sequences differed not only in terms of their duration, but also in terms of the task that participants had to perform to maintain their attention focused. Further research could therefore help to clarify whether these factors may have contributed to the conflicting results obtained in these two studies (Fig. 4).

Another interesting attempt to explore the crossmodal nature of the temporal recalibration process was reported recently by Di Luca, Machulla, and Ernst (2009). In their study, the authors investigated whether, and to what extent, the audiovisual temporal recalibration effect transfers to the perception of simultaneity for visuotactile and audiotactile stimulus pairs. The interesting result to emerge from this experiment was that the transfer of audiovisual recalibration of simultaneity to the other pairings of stimulus modalities was dependent on the location from which the stimuli happened to be presented. Specifically, when the stimuli were presented from the same spatial location in front of participants, the audiovisual recalibration effect transferred to visuotactile stimulus pairs (and not to the audiotactile pairings). By contrast, when the auditory stimuli were presented over headphones instead (i.e., when the auditory stimuli were not colocated with the visual and tactile stimuli), the audiovisual temporal recalibration effect transferred to the audiotactile stimulus pairs (and not to the visuotactile pairings). The fact that audiovisual temporal recalibration differently affected the other two sensory pairings could be due, at least according to Di Luca et al., to the different spatial arrangement of the stimuli. More precisely, in the colocation condition, a change in the perceptual latency (and in the reaction time; RT) of the visual stimuli was observed (see Fig. 5a), whereas in the different location condition, a change in the perceptual latency (and in RT) of the auditory stimuli (see Fig. 4b) was observed instead (cf. Sternberg \& Knoll, 1973).

Di Luca et al. (2009) argued that the repeated exposure to asynchronous audiovisual stimuli gave rise to the adjustment of the perceptual latency of, separately, the visual or the auditory stimulus. This, in turn, caused a recalibration of perceived simultaneity within the stimulus pairings which, as in Di Luca et al.'s study, were different from those composing the stimuli repeatedly presented during the exposure phase of their experiment. Moreover, the mode of presentation has been shown to affect which signal estimate is trusted more, and hence which signal undergoes a change of temporal latency during recalibration. In Di Luca et al.'s study, when the auditory stimuli were presented over headphones (which constitutes a nonfixed external stimulus source, since it moves when the head moves), the auditory estimate was more likely to be biased (and thus trusted less) than the visual estimate. As a consequence, in this condition, it is the auditory estimate that is temporally recalibrated toward the visual standard. This is seen as an increased transfer to the perception of audiotactile simultaneity as compared with the perception of visuotactile simultaneity. By contrast, when the auditory stimuli were spatially fixed, the visual estimate was more likely to be biased, thus giving rise to an opposite pattern of results (Di Luca et al., 2009).

The fact that temporal recalibration transfers across sensory modality (see also Nagarajan, Blake, Wright, Byl, \& Merzenich, 1998, for a study investigating the transfer of temporal rate discrimination from touch to audition) leads on to the crucial question of whether the extraction of temporal rate information should be considered as being coupled to a 
Exposed to sound / light
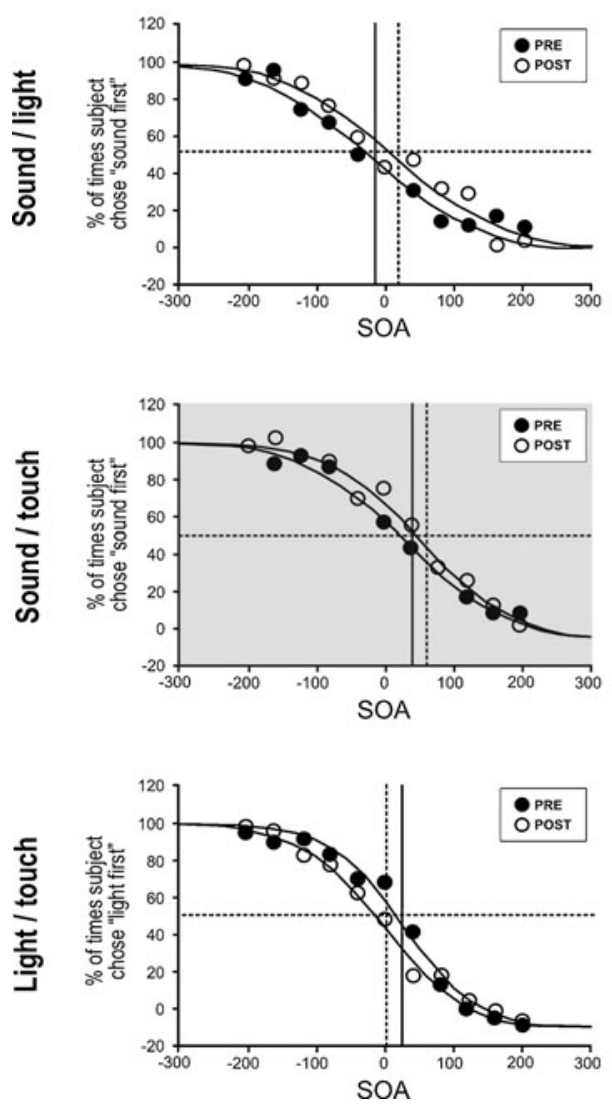

Exposed to sound / touch
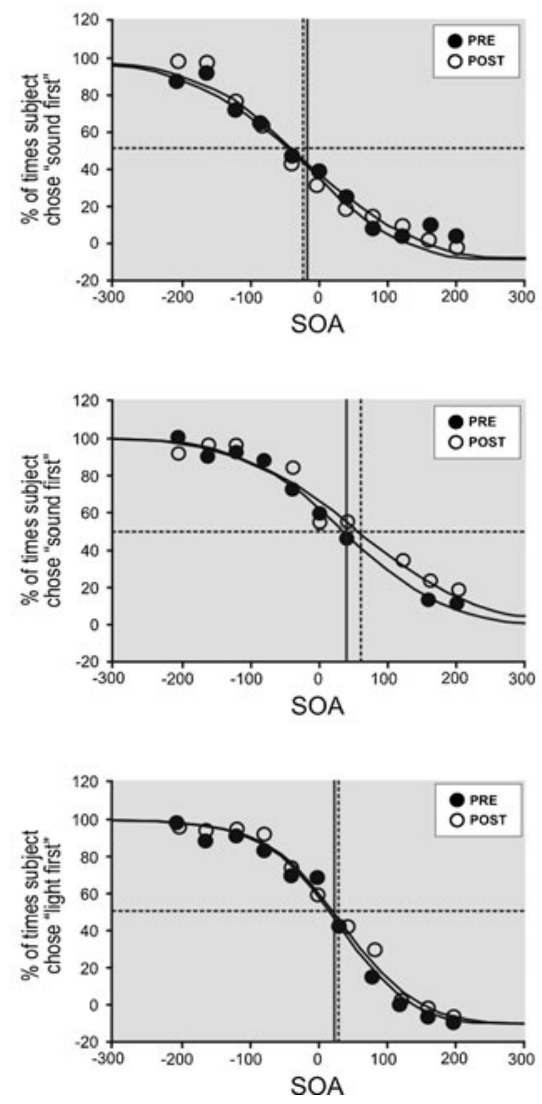

Exposed to light / touch
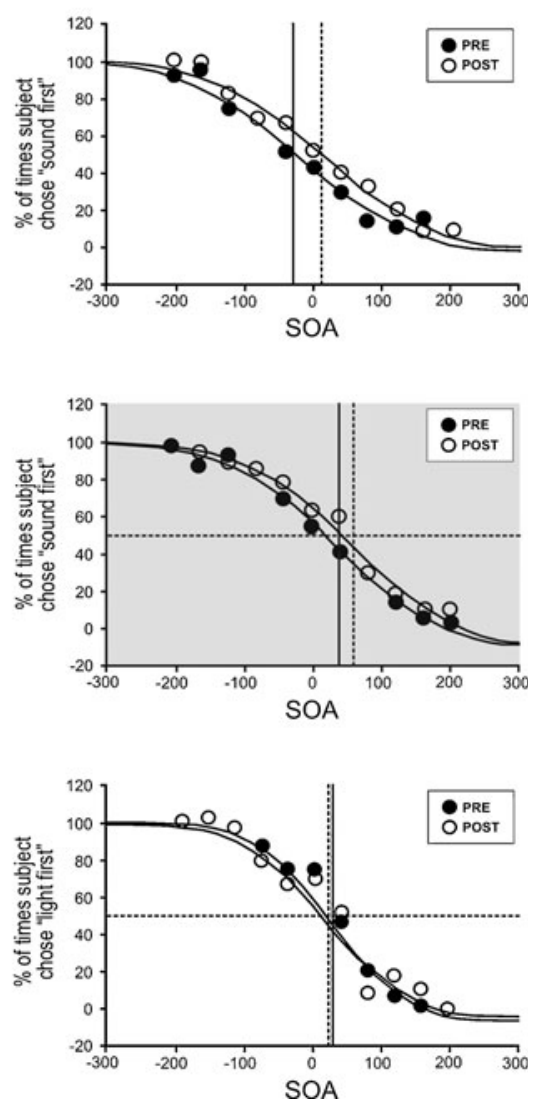

Fig. 4 Average cumulative Gaussian curves before and after exposure to time-staggered bimodal stimulus pairs. The solid vertical line and filled black symbols represent responses in the preexposure phase. The dotted vertical line and open symbols are postexposure. The three columns are arranged according to the stimulus pair used in the exposure phase: sound/light (light leading by $100 \mathrm{~ms}$ ), sound/touch (touch leading by

sensory modality or whether instead it is represented amodally (e.g., see Grondin, 2010; van Wassenhove, 2009; Wittmann, 2009). Despite the remarkable body of evidence accumulated on this topic, the large degree of inconsistency,
$100 \mathrm{~ms}$ ), and light/touch (touch leading by $100 \mathrm{~ms}$ ), respectively. The rows are arranged according to the stimulus pairs tested: sound/light (positive means "light first"), sound/touch (positive means "touch first"), light/touch (positive means "touch first"). The three shaded graphs are the combinations involving auditory and tactile stimuli (modified from Harrar \& Harris, 2008, Fig. 2)

as highlighted by the data reviewed here, seems to suggest that the exact nature of the temporal features that characterize audiotactile interactions are still unresolved, and thus are certainly worthy of further investigation.
Fig. 5 PSS obtained for the three stimulus pairs (i.e., $A V$ audiovisual, $A T$ audiotactile, $V T$ visuotactile) when the auditory stimuli were presented without headphones (a; Experiment 1) or via headphones (b; Experiment 2). Error bars represent the standard error of the mean across participants. Significant effects are indicated by an asterisk $(p<.05$; modified from Di Luca et al., 2009, Figs. 5 and 6)
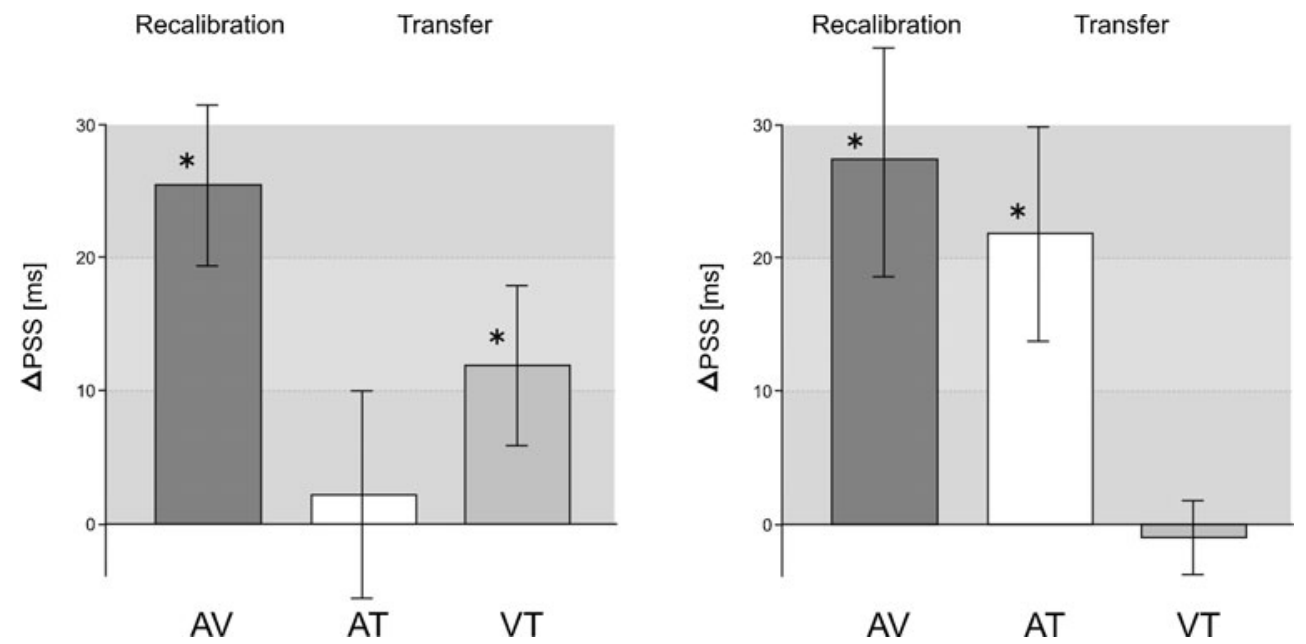


\section{Attention and temporal perception: the prior entry effect}

A number of studies have addressed the question of whether temporal perception and, in particular, the impression of temporal simultaneity/successiveness can be influenced by the attentional focus of participants. One of the best-studied attentional effects on temporal perception is the phenomenon of "prior entry" (see Spence \& Parise, 2010, for a recent review). According to the law of prior entry (Titchener, 1908), the attended stimuli (or modality) will be perceived sooner than when attention is focused elsewhere (or on another modality). This effect has traditionally been assessed by means of the aforementioned TOJ task, and is measured as a significant difference in the PSS between conditions in which one of the target stimuli is attended as compared with when the other stimulus is attended instead (or when attention is divided).

Studies investigating the audiotactile prior entry effect have demonstrated that the endogenous focusing of an observer's attention on one sensory modality can effectively modulate the perceived temporal relationship between pairs of stimuli (Sternberg, Knoll, \& Gates, 1971; Stone, 1926; see also Sternberg \& Knoll, 1973; Van Damme, Gallace, Spence, Crombez, \& Moseley, 2009). Moreover, the focusing of a person's attention on a specific location or sensory modality, as well as toward a particular point in time, determines the relative speeding up in the processing of the auditory and tactile stimuli, as shown by Lange and Röder (2006). In their study, participants were presented with short (600 ms) and long (1,200 ms) empty intervals, marked by a tactile onset and an auditory or tactile offset marker (which could consist in a continous stimulation, or in a stimulation with a gap), and, on a block-by-block basis, were asked to attend to one interval and to one sensory modality. The participants had to decide as quickly and as accurately as possible whether the offset marker was a single or a double stimulus. The behavioral and electrophysiological results of this study demonstrated that focusing attention on a particular point in time facilitated the processing of auditory and tactile stimuli. More specifically, participants responded more rapidly to stimuli at an attended point in time as compared with stimuli that were relatively less attended, irrespective of which modality was task relevant. Moreover, an enhancement of early negative deflections of the auditory and somatosensory eventrelated potentials (ERPs; for audition, 100-140 ms; for touch, 130-180 ms) were observed when audition or touch were task relevant, respectively.

These results therefore suggest that the allocation of attention along the temporal dimension can affect the early stages of sensory processing. More interestingly, these data also demonstrate that focusing attention on a particular point in time results in the more efficient processing of stimuli presented in both audition and touch. In contrast with the results obtained in a sustained attention task, such as that conducted by Lange and Röder (2006), no modulation of sensory processing by temporal attention has been detected in a visual temporal cuing paradigm (cf. Griffin, Miniussi, \& Nobre, 2001; Miniussi, Wilding, Coull, \& Nobre, 1999). Lange and Röder suggested that this discrepancy between the effects seen for different modalities could be attributed to the fact that a sustained attention paradigm, such as that used in their study, could have facilitated a more stable representation of the time interval to be attended to. In this regard, their paradigm differs from the conditions in which the to-be-attended time point changes on a trial-by-trial basis (as in cuing paradigms). This, in turn, could have favored the emergence of earlier effects of temporal attention observed in their study. According to an alternative account for these data, the temporal acuity of audition and touch is simply higher than that of vision (see also Fujisaki \& Nishida, 2009).

\section{Numerosity}

The decision to review those studies that have investigated numerosity judgments in the present review is supported by the functional link that exists between time and numerosity processing, as first proposed by Meck and Church (1983). In a series of experiments, these researchers demonstrated that rats automatically process duration and number when these two dimensions covary in a sequence of events (i.e., rats were equally sensitive to a $4: 1$ ratio for both counts and durations, with the other dimension being controlled; see also Brannon, Suanda, \& Libertus, 2007, for evidence in children), suggesting that there is a similarity between the processes underlying counting and timing. Interestingly, this effect, which was first demonstrated with auditory inputs, has now been generalized to the case of cutaneous signals. Meck and Church proposed the existence of a single shared representational mechanism (i.e., an "internal accumulator") that supervises both the duration and the numerosity of the events (and, for some authors, their spatial location; see Cappelletti, Freeman, \& Cipolotti, 2009; Walsh, 2003).

In a typical temporal numerosity judgment task, a sequence of stimuli (i.e., flashes, beeps, or taps) is presented, and the observer has to try and judge how many stimuli have been presented (e.g., see Cheatham \& White, 1952, 1954; Taubman, 1950a, 1950b). The first study to have compared the ability of participants to perform the tactile, visual, and auditory temporal numerosity discrimination of trains of stimuli (consisting of two to nine pulses) presented from a single location at different rates (varying from three to eight pulses per second) was conducted by 
Lechelt (1975). His results demonstrated that there was a generalized tendency toward the underestimation of the number of pulses, and the number of errors in number assessment was more pronounced as the number of pulses and/or the rate of stimulus presentation increased. More interestingly, in the context of the present review, was his finding that modality-specific differences were also observed. In all of the experimental conditions, the accuracy in the temporal numerosity judgment task was found to be much higher for audition than for either touch or vision.

A recent study investigated whether the combination of trains of stimuli presented simultaneously in more than one sensory modality could improve people's temporal numerosity estimates (Philippi, van Erp, \& Werkhoven, 2008). In contrast with other studies (e.g., Bresciani \& Ernst, 2007; Bresciani et al., 2005; Hötting \& Röder, 2004; Shams, Kamitani, \& Shimojo, 2000) that explored whether there was any interfering effect between sequences of stimuli, the goal of Philippi et al.'s study was to explore whether the presentation of congruent sequences of stimuli would have a beneficial effect on participants' temporal numerosity estimation judgments (see also Lee \& Spence, 2008, 2009). The participants in this particular study were presented with sequences (i.e., 2 to 10) of stimuli at interstimulus intervals (ISIs) varying from 20 to $320 \mathrm{~ms}$. The participants were instructed to use the multisensorially redundant information to their advantage when performing the task. Indeed, the results revealed that the degree of underestimation (which has been consistently found in previous unisensory temporal numerosity estimation judgment studies; e.g., Lechelt, 1975; White \& Cheatham, 1959) and the variance in participants' estimates were reduced as compared with those in the conditions of unisensory stimulus presentation. The results of Philippi et al.'s study confirmed that visual judgments were worse than the auditory judgments, and that these, in turn, were worse than the tactile judgments (see Lechelt, 1975; White \& Cheatham, 1959). Overall, the results showed that the underestimation decreased for smaller ISIs (i.e., 20 and $40 \mathrm{~ms}$ ) in the multisensory as compared with the unisensory presentation conditions. The authors explained this result by considering that the persistence of brief stimuli hampers the clear separation between rapidly presented within-modality signals, even leading to their fusion, under conditions in which the ISIs are much smaller than the persistence of each individual signal. Of interest for present purposes, Philippi et al. (2008) observed that the difference in temporal numerosity estimation judgments between unimodal auditory or tactile conditions and the bimodal audiotactile condition differed significantly only for short ISIs (20 and $40 \mathrm{~ms}$ ).

As already mentioned, a large number of previous studies have investigated whether, and to what extent, the presentation of incongruent task-irrelevant multisensory sequences of pulses can influence people's temporal numerosity judgments in the target modality. In the illusory flash paradigm, for instance, people are instructed to report the number of flashes presented with to-be-ignored incongruent sequences of beeps (Shams et al., 2000). The striking result to have emerged from this study was that, when presented with a single flash and multiple auditory pulses, observers typically perceived an illusory second flash. This illusory effect has been explained by taking into account the higher reliability of the auditory modality as compared with the visual modality in the temporal domain (see Shams, Ma, \& Beierholm, 2005). This effect, which constitutes a robust perceptual phenomenon, has now been replicated in the audiotactile not to mention visuotactile domains (Bresciani et al., 2005; Bresciani \& Ernst, 2007; Hötting, Friedrich, \& Röder, 2009; Hötting \& Röder, 2004). In one of their studies, Bresciani and Ernst (2007) presented series of beeps and taps and had their participants report on the number of tactile stimuli while ignoring the auditory distractors. The results revealed that participants' tactile perception was modulated by the presentation of taskirrelevant auditory stimuli, with their responses being significantly affected by the number of beeps that were delivered. Such a modulation only occurred, however, when the auditory and tactile stimuli were similar enough (i.e., had the same duration) and were presented at around the same time (see Fig. 6).

According to the maximum likelihood estimation model of multisensory integration (e.g., Alais, Newell, \& Mamassian, 2010), the reliability of a sensory channel is related to the relative uncertainty of the information it conveys. The higher the relative variance of a sensory modality, the weaker its relative reliability (Ernst \& Bülthoff, 2004). In another study, in order to investigate whether the auditory bias on tactile perception could be disrupted by manipulating the reliability of the auditory information, Bresciani and Ernst (2007) varied the intensity of the beeps. The auditory stimuli were presented at either 41 or $74 \mathrm{~dB}$ (signal-to-noise ratio of, respectively, -30 and $3 \mathrm{~dB}$ ). They found that the participants were more sensitive (i.e., their estimates were less variable) in counting the number of the more intense (rather than the less intense) beeps presented with the irrelevant taps. Conversely, they were more sensitive in counting the number of taps presented with less intense (vs. more intense) irrelevant beeps (see also Wozny, Beierholm, \& Shams, 2008). This pattern of results could reflect the fact that the decrease in the intensity of the auditory stimuli reduced the relative reliability of the auditory modality, thus inducing differential interactions with touch as a function of the intensity of the stimuli. Taken together, these results therefore show that audition and touch reciprocally bias each 


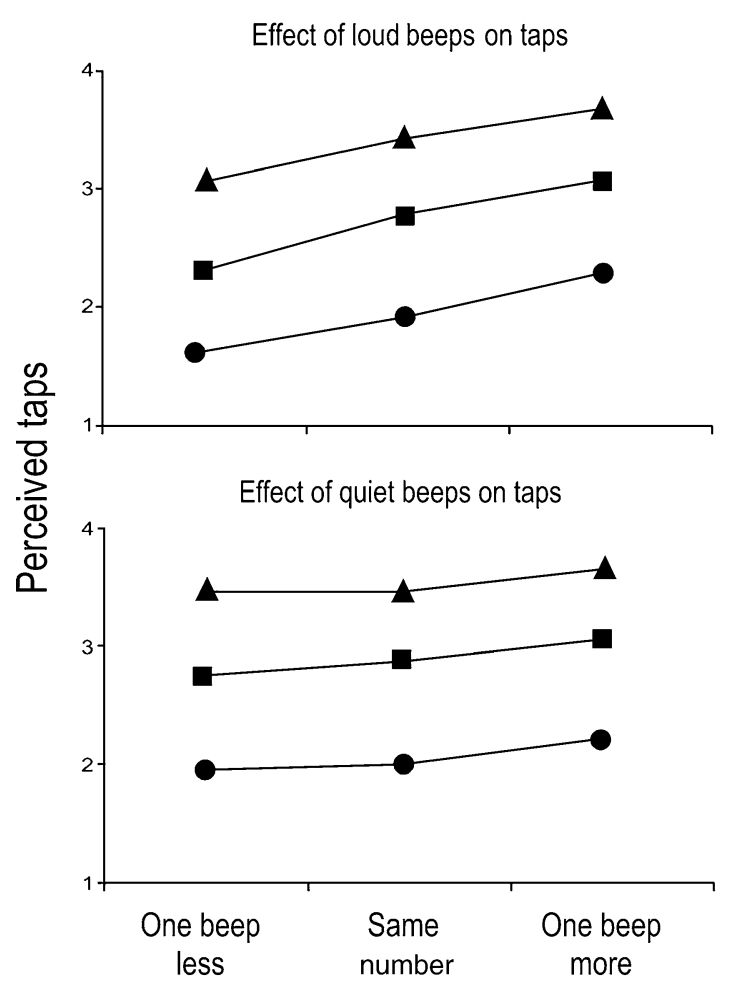

\section{Background condition}

Fig. 6 Number of perceived events in the target modality as a function of both the actual number of events delivered (circle for two, square for three, and triangle for four events) and the background condition. The two graphs on the left correspond to the sessions in which the target modality was touch (i.e., the participants had to count the number of taps), and the two graphs on the right correspond to the

other (when alternatively used as target or distractor), with the degree of evoked bias depending on the relative reliability of the two modalities (see also Bresciani, Dammeier, \& Ernst, 2008; Occelli, Spence, \& Zampini, 2009).

To the best of our knowledge, however, the experimental investigations published to date have not applied a multisensory perspective to the assessment of the interactions between time and numerosity (and, possibly, space). Indeed, the currently available data refer to the visual (e.g., Dormal, Seron, \& Pesenti, 2006) or auditory (Droit-Volet, Clement, \& Favol, 2003; Xuan, Zhang, He, \& Chen, 2007) modality, and typically report that when participants perform a numerosity judgment task, temporal intervals are perceived as shorter than their veridical duration. The investigation of this topic using a multisensory approach could lead to a better understanding of the interplay occurring between the mental representation of time, space, numberand, in general, of magnitudes - in both neurologically intact

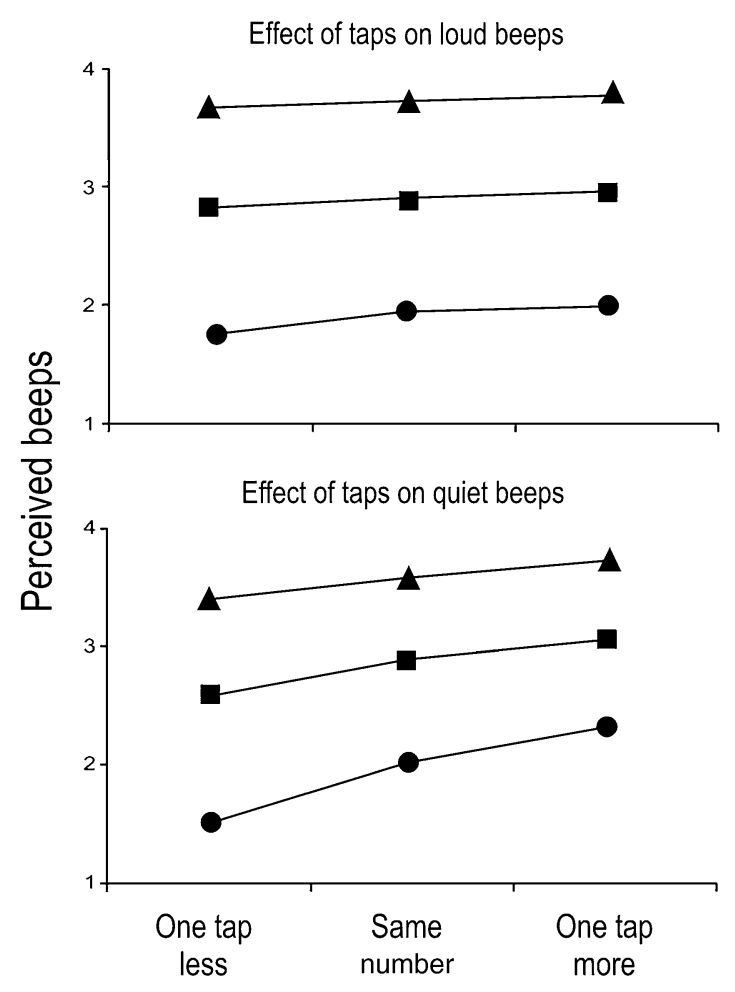

Events presented in the target modality:

- 2 घ $3 \Delta 4$

ffect of taps on loud beeps

sessions in which the target modality was audition (i.e., the participants had to count the number of beeps). The two upper graphs correspond to the sessions in which the beeps were louder, and the two lower graphs to the sessions in which the beeps were quieter (modified from Bresciani \& Ernst, 2007, Fig. 2)

individuals and ultimately in those patients suffering from parietal lesions, a topic which has been, to our knowledge, by now investigated primarily in the visual modality (e.g., Oliveri et al., 2008, 2009; Vicario, Pecoraro, Turriziani, Koch, \& Oliveri, 2008; see also Bueti \& Walsh, 2009).

\section{Audiotactile interactions based on frequency similarity}

Perceptual interactions between hearing and touch are distinctive from those associations occurring between other pairings of sensory modalities (Gescheider, 1970; Soto-Faraco \& Deco, 2009; von Békésy;, 1959; Zmigrod, Spapé, \& Hommel, 2009, Experiment 2). As was already mentioned, auditory and vibrotactile stimuli are transduced by the same physical mechanism (i.e., mechanoreception), consisting of the mechanical stimulation of, respectively, the basilar membrane and the skin. Hence, both auditory and vibrotactile 
stimuli can be described according to their specific periodic patterns of stimulation (i.e., their frequency), defined as the number of repetitions of the sound waveforms (see Plack, 2004; Siebert, 1970) or of tactile pulses (see Luna, Hernández, Brody, \& Romo, 2005), respectively, per unit time. Hence, it seems somehow surprising that the investigation of audiotactile interactions as a function of the frequency similarity between stimuli has rarely been carried out to date. Thus far, the focus has primarily been on the perception of lingual vibrotactile stimulation (Fucci, Petrosino, Harris, \& Randolph-Tyler, 1988; Harris, Fucci, \& Petrosino, 1986, 1989).

The ability of mammals to discriminate frequencies has been considered as reflecting the frequency resolution characterizing the auditory pathway at both the peripheral (i.e., the basilar membrane of the cochlea; Robles \& Ruggero, 2001) and central (i.e., the primary auditory cortex; Langers, Backes, \& van Dijk, 2007; Tramo, Cariani, Koh, Makris, \& Braida, 2005) stages of auditory information processing. The systematic spatial mapping of frequency coding in the brain (known as tonotopy) and the filtering properties of auditory neurons and sensory receptors have been considered responsible for decoding the frequency of auditory stimulation (see Schreiner, Read, \& Sutter, 2000, for a review; see also Elhilali, Ma, Micheyl, Oxenham, \& Shamma, 2009; Romani, Williamson, \& Kaufman, 1982; Schnupp \& King, 2008). However, the tonotopic structure of the auditory system is not the only candidate for the representation of the temporal characteristics of auditory stimuli.

Indeed, the activity of neurons at different stages of the auditory pathway has been shown to change as a function of the repetition rates of the auditory events being processed (see Bendor \& Wang, 2007, for a review). More specifically, acoustic signals within the flutter range (10$45 \mathrm{~Hz}$ ) are coded by neurons that synchronize their activity to the temporal profile of repetitive signals. These neurons have been observed both along the auditory-nerve fibers and in the inferior colliculus, the medial geniculate body, and in a specific neuronal population along the anterolateral border of the primary auditory cortex (AI; Dicke, Ewert, Dau, \& Kollmeier, 2007; Oshurkova, Scheich, \& Brosch, 2008; Wang et al., 2008). Other mechanisms regulate the activity of the neural population coding for auditory signals presented at higher repetition rates (i.e., above the perceptual flutter range). These neurons modify their discharge rates - not their spike timing-as a function of the frequency of the auditory events that are being processed (Oshurkova et al., 2008; Wang et al., 2008). Thus, the temporal profile of auditory stimuli appears to be represented in AI by a dual process (i.e., stimulus-synchronized firing pattern and discharge rate), each involving specific subpopulations of neurons.
The distinct neural encoding of auditory stimuli differing in frequency may also be responsible for the different perceptual impression conveyed by auditory stimuli. Indeed, when auditory events are presented at rates within the $10-45 \mathrm{~Hz}$ (i.e., flutter) range, the resulting percepts tend to consist of sequential and discrete sounds (i.e., acoustic flutter; Bendor \& Wang, 2007; see also Besser, 1967). According to Bendor and Wang, the discrete impression of the flutter percept could be considered as the direct outcome of the synchronized responses representing the event at different neural stages of the auditory pathway. On the other hand, neurons encoding stimuli with repetition rates beyond this range do not synchronize with the stimuli, thus failing to induce the impression of discrete auditory events and instead giving rise to continuous-sounding percepts that have a specific pitch (Bendor \& Wang, 2007; Hall, Edmondson-Jones, \& Fridriksson, 2006; Tramo et al., 2005; Wang et al., 2008).

Interestingly, the perceptual encoding boundary for repetition rates producing low- and high-frequency stimuli seems to be analogous in both hearing and touch (i.e., $\sim 40$ $50 \mathrm{~Hz}$ ). Just as in hearing, the sensation of flutter in touch is induced by periodic trains of impulses at frequencies between $\sim 5$ and $\sim 40 \mathrm{~Hz}$ (e.g., Romo \& Salinas, 2003), whereas higher repetition rates $(\sim 40-400 \mathrm{~Hz})$ induce a sensation of "vibration/buzzing" (LaMotte \& Mountcastle, 1975; Talbot, Darian-Smith, Kornhuber, \& Mountcastle, 1968). Moreover, in the tactile domain, the identification and discrimination of stimuli differing in their frequency relies on the differential sensitivity of sensory receptors and afferent nerve fibers supplying different portions of the skin (Johansson \& Vallbo, 1979a, 1979b; Morioka \& Griffin, 2005). At the fingertips, the class of fibers classified as fast adapting fibers and the receptors known as "meissner corpuscles" are responsible for the processing of low vibrotactile frequencies (i.e., 5-50 Hz), whereas the pacinian fibers associated with the pacinian receptors are more sensitive to higher vibration frequencies (i.e., higher than $40 \mathrm{~Hz}$; Francis et al., 2000; Iggo \& Muir, 1969; Mahns, Perkins, Sahai, Robinson, \& Rowe, 2006; Morley, Vickery, Stuart, \& Turman, 2007; Talbot et al., 1968; Verrillo, 1985). Animal studies have suggested that one possible candidate for signalling information about the frequency of vibrotactile stimuli is an impulse pattern code, according to which the responses of rapidly adapting afferents are phase locked to the periodicity of the vibrotactile stimulus. The strict correspondence between the temporal features of the vibrotactile stimuli and the impulse patterns have not only been observed in the periphery (i.e., along the sensory nerve fibers), but also in neurons at higher levels along the ascending somatosensory pathway (Hernández, Salinas, García, \& Romo, 1997; 
Mountcastle, Steinmetz, \& Romo, 1990; Salinas, Hernández, Zainos, \& Romo, 2000).

Even though the encoding of the frequency pattern of vibrotactile stimuli involves all of the stations along the somatosensory pathway, it is likely that more sophisticated processes, such as those involving the discrimination of different frequencies, occur more centrally. In primates performing a frequency discrimination task, the patterns of firing evoked in SI neurons by the comparison stimulus (i.e., usually presented second in each pair) are independent of those elicited by the standard stimulus (i.e., the first stimulus in each pair). It thus seems as though SI is an unlikely candidate for the encoding of the difference between the two stimuli (Romo \& Salinas, 2003; Salinas et al., 2000). On the other hand, the fact that the response of neurons in the secondary somatosensory cortex (SII) to the second vibration is affected by the frequency of the first vibration suggests that these neurons contribute significantly to the coding of the frequency difference. Taken together, this evidence therefore suggests that, in primates at least, the perceptual comparison between different frequencies takes place in SII. Subsequent decisional processes are thought to involve the medial premotor cortex in the frontal lobe area, whose neuronal activity significantly covaried with monkeys' perceptual reports (de Lafuente \& Romo, 2005). The similarity of the performance demonstrated by monkeys and humans in detecting and discriminating between stimuli differing in frequency suggests that the neural mechanisms investigated in monkeys may be analogous to those that exist in humans (see Romo \& Salinas, 2003; Salinas et al., 2000; Talbot et al., 1968). In humans, just as in monkeys, frequency discrimination does not rely exclusively on SI, but also involves downstream areas, such as SII and some regions in frontal cortex (Harris, Arabzadeh, Fairhall, Benito, \& Diamond, 2006).

A recent fMRI study investigated which brain areas are involved in the discrimination of vibrotactile frequency in humans (Li Hegner et al., 2007). The participants in this study had to report whether the sequentially presented vibrotactile stimuli had the same or different frequency. The results revealed that an extended region was recruited during the performance of the task. Beyond the areas typically involved in this kind of task (i.e., SI and SII), other areas, such as the superior temporal gyrus, the precentral gyrus, ipsilateral insula, and supplementary motor area were also involved ( $\mathrm{Li}$ Hegner et al., 2007). Interestingly, the superior temporal gyrus is known to mediate interactions between auditory and somatosensory stimuli, in both humans (Foxe et al., 2002; Schroeder et al., 2001) and monkeys (Fu et al., 2003; Kayser, Petkov, Augath, \& Logothetis, 2005). Neurons in the auditory belt areas respond not only to pulsed tactile stimulation, but also to vibratory stimuli, thus suggesting that the auditory association cortex acts as a cortical location of convergence for auditory and tactile inputs during the discrimination of tactile frequency (Iguchi, Hoshi, Nemoto, Taira, \& Hashimoto, 2007; Li Hegner et al., 2007; Schürmann, Caetano, Hlushchuk, Jousmäki, \& Hari, 2006; see also Caetano \& Jousmäki, 2006; Golaszewski et al., 2006). The evidence suggesting that the auditory areas involved in the processing of tactile stimuli are endowed with specific frequency profiles and contribute to the vibrotactile frequency discrimination processes, raises the intriguing possibility of anatomofunctional similarities between those cortical regions devoted to the processing of the periodicity of both vibrotactile and auditory stimulation.

A study by Bendor and Wang (2007) would appear to suggest that these similarities could, in fact, be the case. These authors distinguished between two populations of neurons in the auditory cortex, known as "positive monotonic" and "negative monotonic." respectively. The first population typically increased its firing rate proportional to the increase of the repetition rates of an auditory stimulus, whereas the second population showed the opposite pattern of responding (see also Wang et al., 2008). Interestingly, neurons with positive and negative monotonic tuning to stimulus repetition rate have not only been observed in the auditory cortex, but also in the somatosensory cortex beyond SI (Bendor \& Wang, 2007; Salinas et al., 2000). More specifically, neurons have been detected in SII whose spike rate can be positively or negatively related to the vibrotactile stimulus frequency (Luna et al., 2005; Salinas et al., 2000). The fact that neurons showing positive and negative monotonic tuning to stimulus repetition rate can be observed in both auditory and somatosensory cortices points to a commonality in how these two sensory systems might encode variations in the temporal profile of, respectively, auditory and vibrotactile stimuli (Bendor \& Wang, 2007; Wang et al., 2008; see also Soto-Faraco \& Deco, 2009), possibly indicating a potential neural basis for the discrimination of frequencies delivered crossmodally (see Bendor \& Wang, 2007).

Preliminary evidence from Nagarajan et al. (1998) has suggested that temporal information processing could be governed by common mechanisms across the auditory and tactile sensory systems. In their study, participants were presented with pairs of vibratory pulses and were trained to discriminate the temporal interval separating them. The results not only suggested a decrease in the threshold as a function of training, but also the generalization of the improved interval discrimination to the auditory modality. Even though the generalization was constrained to an auditory base interval that was similar to the one that had been trained in touch, these results are intriguing in suggesting that the coding of temporal intervals could be represented centrally (i.e., shared among different sensory 
modalities; cf. van Wassenhove, 2009, see also Fujisaki \& Nishida, 2010).

Additionally, recent neurophysiological evidence in humans has demonstrated that the discrimination of vibrotactile stimuli can be improved significantly in many people simply by providing auditory feedbackwith the same frequency - after the presentation of the tactile stimulation (Iguchi et al., 2007; see Ro, Hsu, Yasar, Caitlin Elmore, \& Beauchamp, 2009). The investigation of the neural substrates of this effect led to the conclusion that the increase of the perceptual accuracy and the speeding up of the discrimination of the tactile frequencies were subserved by the coactivation of SII and the supratemporal auditory cortices along with upper bank of the superior temporal sulcus. The data suggest that auditory feedback could have induced a complementary processing of tactile information by means of an intervening process of acoustic imagery. The results of this study therefore add weight to previous investigations showing considerable crossmodal convergence in the posterior auditory cortex of not only tactile stimulation (e.g., Foxe et al., 2002; Kayser et al., 2005), but also of vibrotactile stimulation, in both normal hearing (e.g., Caetano \& Jousmäki, 2006; Schürmann et al., 2006) and deaf (Levänen \& Hamdorf, 2001) humans.

On the other hand, Yau et al. (2009) demonstrated that auditory stimuli can interfere with tactile frequencydiscrimination responses. The participants in their study had to perform a two-alternative forced choice task judging which of two vibrotactile stimuli (ranging from 100 to $300 \mathrm{~Hz}$, steps of $40 \mathrm{~Hz}$ ) had the higher frequency. Crucially, the second vibrotactile stimulus was accompanied by an auditory stimulus presented at the same or a different frequency as that of the tactile stimulus. The results revealed a decrement in task performance, but only for auditory distractors in the low frequency range. Interestingly, since the same stimulus proved ineffective in modulating an intensity judgment, and was restricted to the conditions in which the tactile stimulus was presented ator near-the same frequency, this interfering effect is thought to be highly specific across similar frequencies. Moreover, the perceived frequency of the tactile stimulus was pulled toward that of the auditory stimulus (see also Ro et al., 2009). According to Yau et al., these results are consistent with a supramodal representation of the temporal rate of sensory inputs (cf. Fujisaki \& Nishida, 2010; Jones, Poliakoff, \& Wells, 2009; van Wassenhove, 2009; see also Ivry \& Schlerf, 2008, and the section Conclusions and Directions for Future Research of the present review). This assumption has, however, been challenged by findings demonstrating that the "fusion threshold" differs across sensory modalities, being higher for vision (Landis, 1954) than for audition (Exner, 1875; von Békésy, 1936) and touch (von Békésy, 1959, 1963). Future investigations could possibly better assess the multisensory perception of frequency, by investigating, for instance, if and to what extent people are able to match stimuli having comparable temporal rate features within each modality, and crossmodally.

\section{Audiotactile interactions in rhythm perception and sensorimotor synchronization}

Some authors have observed that, whereas the spatial properties of the objects-typically related to visual functions - develop after birth, some temporal properties, such as synchrony, tempo, and rhythm, are experienced during prenatal life, through vestibular, tactile, and auditory stimulation (e.g., Groome et al., 2000; Woodward \& Guidozzi, 1992). The different line of development of the sensory modalities would contribute in generating specific "salience hierarchies" for object/event properties, with the processing of auditory-somatosensory synchrony-such as the one elicited by the maternal heartbeat - being one of the first sensory experiences we humans are exposed to (Lickliter \& Bahrick, 2000). The experimental evidence demonstrates that not only can the fetus perceive synchrony, but also that from the twentieth week of gestation, fetal motor activity exhibits synchrony with maternal heart rate activity (DiPietro et al., 2006). Moreover, it has been demonstrated that the synchrony of activity between mother and fetus experienced during uterine life significantly influences the shaping the infant's capability to perceive the alternating light-dark in the circadian cycle (Tsai, Barnard, Lentz, \& Thomas, 2011), possibly representing a precursor of the mutual responsiveness typifying future social interaction (Reyna \& Pickler, 2009).

Given the synchronization experienced with the mother during gestation, primarily based on biological rhythms such as heartbeat or breathing, it should not come as any surprise to find that the capability to discriminate rhythmic sequences develops very early. In fact, it can be observed by 2 months of age (Trehub \& Hannon, 2006). Moreover, infants show a very early capability to recognize and match rhythms that are experienced via multiple sensory channels (see Bahrick, Flom, \& Lickliter, 2002; Bahrick \& Lickliter, 2004, for evidence on audiovisual rhythm perception). A striking demonstration of this phenomenon comes from a study carried out by Phillips-Silver and Trainor (2005). Infants were trained by being bounced to an ambiguous rhythm (i.e. a rhythm without accents), with half of the infants being bounced on every second beat, and the other half on every third beat. After training, the infants exhibited listening preferences for an auditory version of the rhythm pattern, with accents that matched the way in which they had been 
bounced (i.e., every second or every third beat). Thus, prior motor experience affected the infants' auditory rhythm preferences. Interestingly, the same authors also observed that, in adults, the movement of the body influenced the auditory encoding of an ambiguous musical rhythm, whereas visual information had no such effect (Phillips-Silver \& Trainor, 2005). Thus, it seems that in humans, musical rhythm processing is primarily based on multisensory interactions involving auditory and kinaesthetic systems, which persists for the whole life span (Repp \& Penel, 2004; see also Rubinstein, Giladi, \& Hausdorff, 2002, for rhythmic auditory sensory cuing in the treatment of motor impairment in Parkinson's disease, and Zatorre, Chen, \& Penhune, 2007, for a review on the neural substrates of sensorimotor interactions in music).

Although motor activities often have a somatosensory component, only a few of the studies on this topic have assessed the interactions between auditory and tactile systems in rhythm perception more directly (e.g., Brochard, Touzalin, Després, \& Dufour, 2008; Hatta \& Ejiri, 1989). For instance, Patel, Iversen, Chen, and Repp (2005) showed that humans have no difficulty in synchronizing finger tapping with sound sequences, but failed to do the same with sequences composed of visual stimuli. On the basis of this evidence, Brochard et al. (2008) investigated whether the sensation of regular pulse is exclusively auditory or whether humans can also feel the metric structure within the somesthetic domain. Participants were asked to synchronize their finger tapping to two identical rhythmic sequences, presented via either the auditory or tactile modality (i.e., short tones or light touches of the fingertip). The results showed that participants were able to extract the metric structure from sequences presented via tactile stimulation as easily as when unimodal auditory stimulation was delivered. The finding that meter can be perceived outside of the auditory modality is consistent with the hypothesis that the representation of temporal periodicity is subserved by brain areas whose activity is modality independent. Subsequent studies have extended this suggestion by demonstrating that the neural activity evoked by either an auditory or tactile rhythmic sequence involves those areas that are primarily involved in stimulus prediction, such as the lateral and mesial premotor areas, as well as areas involved in the analysis of the temporal structure of the stimuli, such as prefrontal, occipital, temporal, and cerebellar areas (Bengtsson et al., 2009).

Other research has highlighted the fact that the pattern of common activation during auditory and tactile tapping tasks included both sources that were modality independent and a source that varied with the modality of stimulation (Müller et al., 2008). In the auditory pacing condition, it was localized in the contralateral primary somatosensory cortex, whereas during tactile pacing, it was localized in contralateral posterior parietal cortex. Interestingly, the activity of this third source seems to be involved in the evaluation of the temporal features of the stimulation presented in different sensory modalities. This evidence suggests the intriguing possibility that the processing of sensory inputs with a similar temporal profile is supported by the synchronized oscillatory activities of spatially distributed neuronal population occurring in parallel at different locations in the cortex (Sannita, 2000; see also Engel, König, Kreiter, Gray, \& Singer, 1991). According to this hypothesis, the nonlinear endogenous periodicity of the spontaneous oscillatory activity in the neural system is entrained to an external stimulus. This, in turn, results in the transiently activated neuronal aggregates that are separated in space and are pooled in coherent temporal patterns that are functionally related. Thus, according to the "neural resonance phenomena" view, the temporal patterns of sensory stimulation as well as of musical events are perceived because they are intrinsic to the physics of the neural systems involved in perceiving and responding to that class of percepts (Large \& Snyder, 2009).

The investigation of sensorimotor synchronization raises interesting questions relating to the topic of the present review, such as the issue of the temporal features of the interplay between perception and action. A task that has been considered as providing a suitable model to study the production of a periodic pattern of motor acts (tapping or key pressing) in synchrony with a corresponding pattern of stimuli (typically clicks or tones) is the periodic synchronization task (see Repp, 2005, for a review). In this task, the onset of the finger taps usually lead the stimulus onsets by a certain amount of time, ranging from 20 to $50 \mathrm{~ms}$, giving rise to the commonly known "negative asynchrony" effect. Although the reason behind this effect is not yet completely clear, the explanation that currently has the most support claims that this error could be attributed to differences in the nerve conduction time between click and tap and their corresponding central representations (Paillard-Fraisse hypothesis; Fraisse, 1980; see also Aschersleben, 2002, for a coverage of the explanatory models of this effect). Whereas the majority of studies have investigated synchronization to unimodal (typically auditory) stimulus sequences (e.g., Elliott, Welchman, \& Wing, 2009; Hary \& Moore, 1987; Rivenez, Drake, Brochard, \& Guillaume, 2008), a recent study attempted to assess synchronization to multisensory cues (Elliott, Wing, \& Welchman, 2010). Participants tapped in time with auditory, visual, and tactile metronomic signals, under conditions of either unimodal or bimodal stimulus presentation. The study showed that when rhythmic cues are presented, the brain weights signals according to the relative reliability in the timing of events across modalities, and the movement production is optimally synchronized to the extracted signals. However, the integration between signals 
breaks down when there are large temporal discrepancies between cues. In that case, actions are synchronized with the cue with the highest sensory reliability. Typically, in audiovisual and audiotactile combinations, a bias toward audition is observed. This result indicates that when signals are judged as being independent (temporally separated), asynchrony is computed in relation to one of them (often to the auditory signal; Wing, Doumas, \& Welchman, 2010). Interestingly, according to the results of Elliott et al.'s research (2010), the temporal window of integration between multisensory cues depends on the sensory modalities involved and the likelihood that the stimulation originates from a common source (see Aschersleben, 2002; Aschersleben \& Prinz, 1995). Thus, it seems that our brain is more willing to tolerate a difference in onset time between, for example, a pair of visuotactile stimuli than between a pair of audiotactile stimuli.

Very recent findings in primate studies have shown extensive corticothalamic connections that support the existence of a circuit allowing the rapid integration of somatosensory and auditory signals - processed in remote cortical areas - and the production of a fast motor action to the combination of the two stimuli (see Wang et al., 2008; see also Lim, Bradshaw, Nicholls, \& Altenmüller, 2005). For instance, a restricted thalamic area connects remote cortical areas, such as the auditory cortex, area 5, and auditory belt/parabelt areas with associative parietal areas. This pathway could provide the anatomical support for the rapid transmission of auditory and somatosensory information, converging onto the premotor cortex. (Cappe et al., 2009; see also Hackett et al., 2007).

\section{Conclusions and directions for future research}

Throughout this review, we have illustrated that the auditory-tactile linkage in the temporal domain is special in a number of important ways. The unique interplay between audition and touch is rooted in the common evolutionary origins of these sensory systems and receptors, and evolves into interactions in different domains, which have been described in the previous sections. As can be inferred from these data, the exact nature of the processes governing crossmodal temporal perception is still far from being completely clear, despite the growing number of studies on this topic, which have been reviewed here. In particular, the fact that the temporal processing of crossmodal signals from the auditory and the tactile channels is privileged - or is at least distinguishable - as compared with the other stimulus pairings, given the analogies occurring between the two sensory systems, has so far received partial support (see Fujisaki \& Nishida, 2009). That said, the contradictory evidence on this topic prevents us from providing an unambiguous answer to the question of whether time is represented centrally, in an amodal way, or is rather differently represented according to the modality of the stimuli processed. Furthermore, we are aware that some of the dimensions regarding the perception of time - such as time-duration perception - have not been covered in this review. However, rather surprisingly, it turns out that these issues have not been investigated extensively thus far in the audiotactile domain. Indeed, playing a musical instrument is a rather complex task. It requires fine temporal accuracy skills (i.e., synchronization, proficient temporal rate, and rhythm processing and reproduction) and the highly proficient coordination of motor activities (closely related with somatosensory and proprioceptive feedback; Drost, Rieger, Brass, Gunter, \& Prinz, 2005; Goebl \& Palmer, 2008; Huang, Gamble, Wang, \& Hsiao, 2010) with their auditory outcomes. Although the consequences of musical training on audiotactile interactions have been explored at the neuronal level (e.g., Lappe, Herholz, Trainor, \& Pantev, 2008; Lotze, Scheler, Tan, \& Braun, \& Birbaumer, 2003; Pantev, Lappe, Herholz, \& Trainor, 2009; Pantev et al., 2003; Popescu, Otsuka, \& Ioannides, 2004; Schulz, Ross, \& Pantev, 2003), there is little behavioral evidence (though see Müller et al., 2008; Pollok, Müller, Aschersleben, Schnitzler, \& Prinz, 2004).

Another dimension that is related to musical perception is temporal perception, in particular, the perception of temporal duration which, although extensively studied in the audiovisual domain (e.g., Burr, Banks, \& Morrone, 2009; Ulrich, Nitschke, \& Rammsayer, 2006; van Wassenhove, Buonomano, Shimojo, \& Shams, 2008; Vicario, Rappo, Pepi, \& Oliveri, 2009), has rarely been studied in the other sensory domains (though see Goodfellow, 1934; Schutz \& Kubovy, 2009). Very recently, however, an EEG study compared the ability of sighted and blind people to discriminate the temporal duration of auditory and tactile stimuli (see van der Lubbe, Van Mierlo, \& Postma, 2010). Separate tactile and auditory duration discrimination tasks were conducted, with participants instructed to recognize auditory or tactile targets that were preceded by to-be-ignored tactile or auditory cues to the same or different side as the targets (i.e., valid or invalid trials). The results revealed that the blind had superior duration discrimination abilities as compared with sighted controls. This difference was demonstrated in both the speed and accuracy of their responses. Their proficient performance was accompanied by an enlarged posterior negativity (relative to that seen in the sighted), in both tactile and auditory tasks. Since no reduced orienting effects induced by crossmodal cues were observed in the blind, it seems that changes in information processing in the early blind participants are not attributable to modifications in structures relevant for attentional orienting and alertness, but to a later-supramodal-level of processing instead.

Taken together, these findings support the hypothesis that the enhanced performance and the pattern of activations observed in the blind could reflect a modification at a higher 
level of information processing, instead of changes in early perceptual processes within the occipital cortex. Furthermore, these data are in line with an amodal, rather than a modality specific, temporal perception process that involves occipital areas in the blind. From these preliminary results, investigating audition and touch separately, it would appear that duration perception in these two sensory modalities could also represent a promising field to be investigated more thoroughly in future research, especially in the clinical field (Meck, 2005). A further issue worthy of investigation could be to find out whether the perception of the duration of auditory and tactile stimuli is prone to crossmodal distortion effects, analogous to what has been found between auditory and visual stimuli (e.g., Chen \& Yeh, 2009; van Wassenhove et al., 2008). Moreover, the potential implementation of the experimental findings in the clinical field, such as in sensory substitution (e.g., Minagawa, Ohnishi, \& Sugie, 1996), in the diagnosis (e.g., Danckert et al., 2007; Davalos, Kisley, \& Ross, 2002; Gilden \& Marusich, 2009; Smith, Harper, Gittings, \& Abernethy, 2007), or in the treatment (Powers, Hillock, \& Wallace, 2009; Thaut, 2005; Vargas \& Yu, 2008) of psychiatric and neurological diseases, would be an interesting issue to be investigated in future studies.

Lastly, and as pointed out recently by van Wassenhove (2009), sensory illusions could also offer cues into the conditions under which the perception of the real can be turned into distorted percepts (mainly, but not exclusively, of a spatial nature). Indeed, in this regard, the temporal characteristics of stimulation seem to play a very important role in determining, for instance, the emergence of perceptual illusions (Eagleman, 2008; Grush, 2005), crossmodally (e.g., as in the parchment skin illusion; Guest, Catmur, Lloyd, \& Spence, 2002; Jousmäki \& Hari, 1998; and auditory-tactile crossmodal saltation; Trojan, Getzmann, Möller, Kleinböhl, \& Hölzl, 2009), or in touch and hearing separately (e.g., as in the funnelling illusion, Chen, Friedman, \& Roe, 2003; Gardner \& Spencer, 1972; Sherrick, 1964; von Békésy, 1959, 1967; Watanabe, 1979; saltation; Geldard, 1985; Hari, 1995; see also Geldard \& Sherrick, 1972; and chronostasis; Hodinott-Hill, Thilo, Cowey, \& Walsh, 2002; Yarrow \& Rothwell, 2003). The discovery of new perceptual illusory phenomena could, in the future, provide clues into the temporal features governing audiotactile interactions.

Author note We thank Paola Rigo and Tommaso Sega for preparing the figures. V.O. is supported by a Departmental Fellowship from the Department of Cognitive Sciences and Education, University of Trento.

\section{References}

Adelstein, B. D., Begault, D. R., Anderson, M. R., \& Wenzel, E. M. (2003). Sensitivity to haptic-audio asynchrony. In Sharon L.
Oviatt, Trevor Darrell, Mark T. Maybury, Wolfgang Wahlster (Eds.), Proceedings of the 5th International Conference on Multimodal Interfaces, 5-7th November 2003 (pp. 73-76). Vancouver, British Columbia, Canada: ACM New York, NY, USA.

Alais, D., Newell, F. N., \& Mamassian, P. (2010). Multisensory processing in review: From physiology to behaviour. Seeing and Perceiving, 23, 3-38.

Aschersleben, G. (2002). Temporal control of movements in sensorimotor synchronization. Brain and Cognition, 48, 66-79.

Aschersleben, G., \& Prinz, W. (1995). Synchronizing actions with events: The role of sensory information. Perception \& Psychophysics, 57, 305-317.

Bahrick, L. E., Flom, R., \& Lickliter, R. (2002). Intersensory redundancy facilitates discrimination of tempo in 3-month-old infants. Developmental Psychobiology, 41, 352-363.

Bahrick, L. E., \& Lickliter, R. (2004). Infants' perception of rhythm and tempo in unimodal and multimodal stimulation: A developmental test of the intersensory redundancy hypothesis. Cognitive, Affective, \& Behavioral Neuroscience, 4, 137-147.

Beauchamp, M. S. (2005). Statistical criteria in fMRI studies of multisensory integration. Neuroinformatics, 3, 93-113.

Begault, D. R., Adelstein, B. D., McClain, B. U., \& Anderson, M. R. (2005). Thresholds for auditory-tactile asynchrony. Conference paper 6511. In Proceedings of the Audio Engineering Society 118th Convention, 28-31 May 2005. Barcelona, Spain: Audio Engineering Society Preprints.

Bendor, D., \& Wang, X. (2007). Differential neural coding of acoustic flutter within primate auditory cortex. Nature Neuroscience, 10, $763-771$

Bengtsson, S. L., Ullén, F., Ehrsson, H. H., Hashimoto, T., Kito, T., Naito, E., et al. (2009). Listening to rhythms activates motor and premotor cortices. Cortex, 45, 62-71.

Besser, G. M. (1967). Some physiological characteristics of auditory flutter fusion in man. Nature, 214, 17-19.

Bleckmann, H. (2008). Peripheral and central processing of lateral line information. Journal of Comparative Physiology. A, Neuroethology, Sensory, Neural, and Behavioral Physiology, 194, 145-158.

Brannon, E. M., Suanda, S., \& Libertus, K. (2007). Temporal discrimination increases in precision over development and parallels the development of numerosity discrimination. Developmental Science, 10, 770-777.

Bresciani, J. P., Dammeier, F., \& Ernst, M. O. (2008). Tri-modal integration of visual, tactile and auditory signals for the perception of sequences of events. Brain Research Bulletin, 75, 753-760.

Bresciani, J., \& Ernst, M. O. (2007). Signal reliability modulates auditorytactile integration for event counting. Neuroreport, 18, 1157-1161.

Bresciani, J. P., Ernst, M. O., Drewing, K., Bouyer, G., Maury, V., \& Kheddar, A. (2005). Feeling what you hear: Auditory signals can modulate tactile tap perception. Experimental Brain Research, $162,172-180$.

Brochard, R., Touzalin, P., Després, O., \& Dufour, A. (2008). Evidence of beat perception via purely tactile stimulation. Brain Research, 1223, 59-64.

Bueti, D., \& Walsh, V. (2009). The parietal cortex and the representation of time, space, number and other magnitudes. Philosophical Transactions of the Royal Society of London. Series B, Biological Sciences, 364, 1831-1840.

Burr, D., Banks, M. S., \& Morrone, M. C. (2009). Auditory dominance over vision in the perception of interval duration. Experimental Brain Research, 198, 49-57.

Caetano, G., \& Jousmäki, V. (2006). Evidence of vibrotactile input to human auditory cortex. NeuroImage, 29, 15-28.

Calvert, G. A., Spence, C., \& Stein, B. E. (Eds.). (2004). The handbook of multisensory processes. Cambridge: MIT Press. 
Cappe, C., Morel, A., Barone, P., \& Rouiller, E. M. (2009). The thalamocortical projection systems in primates: An anatomical support for multisensory and sensorimotor interplay. Cerebral Cortex, 19, 2025-2037.

Cappelletti, M., Freeman, E. D., \& Cipolotti, L. (2009). Dissociations and interactions between time, numerosity and space processing. Neuropsychologia, 47, 2732-2748.

Cheatham, P. G., \& White, C. T. (1952). Temporal numerosity: I. Perceived number as a function of flash number and rate. Journal of Experimental Psychology, 44, 283-287.

Cheatham, P. G., \& White, C. T. (1954). Temporal numerosity: III. Auditory perception of number. Journal of Experimental Psychology, 47, 425-428.

Chen, L. M., Friedman, R. M., \& Roe, A. W. (2003). Optical imaging of a tactile illusion in area $3 \mathrm{~b}$ of the primary somatosensory cortex. Science, 302, 881-885.

Chen, K. M., \& Yeh, S. L. (2009). Asymmetric cross-modal effects in time perception. Acta Psychologica, 130, 225-234.

Collignon, O., Renier, L., Bruyer, R., Tranduy, D., \& Veraart, C. (2006). Improved selective and divided spatial attention in early blind subjects. Brain Research, 1075, 175-182.

Corey, D. P. (2003). New TRP channels in hearing and mechanosensation. Neuron, 39, 585-588.

Crystal, J. D. (2009). Theoretical and conceptual issues in time-place discrimination. European Journal of Neuroscience, 30, 17561766.

Danckert, J., Ferber, S., Pun, C., Broderick, C., Striemer, C., Rock, S., et al. (2007). Neglected time: Impaired temporal perception of multisecond intervals in unilateral neglect. Journal of Cognitive Neuroscience, 19, 1706-1720.

Davalos, D. B., Kisley, M. A., \& Ross, R. G. (2002). Deficits in auditory and visual temporal perception in schizophrenia. Cognitive Neuropsychiatry, 7, 273-282.

de Lafuente, V., \& Romo, R. (2005). Neuronal correlates of subjective sensory experience. Nature Neuroscience, 8, 1698-1703.

D'Elia, A., Pighetti, M., Vanacore, F., Fabbrocini, G., \& Arpaia, L. (2005). Vibroacoustic stimulation in normal term human pregnancy. Early Human Development, 81, 449-453.

Di Luca, M., Machulla, T. K., \& Ernst, M. O. (2009). Recalibration of multisensory simultaneity: Cross-modal transfer coincides with a change in perceptual latency. Journal of Vision, 9, 1-16.

Dicke, U., Ewert, S. D., Dau, T., \& Kollmeier, B. (2007). A neural circuit transforming temporal periodicity information into a ratebased representation in the mammalian auditory system. Journal of the Acoustical Society of America, 121, 310-326.

DiPietro, J. A., Caulfield, L. E., Irizarry, R. A., Chen, P., Merialdi, M., \& Zavaleta, N. (2006). Prenatal development of intrafetal and maternal-fetal synchrony. Behavioral Neuroscience, 120, 687-701.

Dormal, V., Seron, X., \& Pesenti, M. (2006). Numerosity-duration interference: A Stroop experiment. Acta Psychologica, 121, 109124.

Droit-Volet, S., Clement, A., \& Fayol, M. (2003). Time and number discrimination in a bisection task with a sequence of stimuli: A developmental approach. Journal of Experimental Child Psychology, 84, 63-76.

Droit-Volet, S., \& Gil, S. (2009). The time-emotion paradox. Philosophical Transactions of the Royal Society of London. Series B, Biological Sciences, 364, 1943-1953.

Drost, U. C., Rieger, M., Brass, M., Gunter, T. C., \& Prinz, W. (2005). When hearing turns into playing: Movement induction by auditory stimuli in pianists. Quarterly Journal of Experimental Psychology, 58A, 1376-1389.

Eagleman, D. M. (2008). Human time perception and its illusions. Current Opinion in Neurobiology, 18, 131-136.

Eimer, M. (2004). Multisensory integration: How visual experience shapes spatial perception. Current Biology, 14, R115-R117.
Elhilali, M., Ma, L., Micheyl, C., Oxenham, A. J., \& Shamma, S. A. (2009). Temporal coherence in the perceptual organization and cortical representation of auditory scenes. Neuron, 61, 317-329.

Elliott, M., Welchman, A. E., \& Wing, A. M. (2009). Being discrete helps keep to the beat. Experimental Brain Research, 192, 731-737.

Elliott, M. T., Wing, A. M., \& Welchman, A. E. (2010). Multisensory cues improve sensorimotor synchronisation. European Journal of Neuroscience, 31, 1828-1835.

Engel, A. K., König, P., Kreiter, A. K., Gray, C. M., \& Singer, W. (1991). Temporal coding by coherent oscillations as a potential solution to the binding problem: Physiological evidence. In H. G. Shuster (Ed.), Nonlinear dynamics and neuronal networks (pp. 3-25). Weinheim: VCH Verlagsgesellschaft.

Ernst, M. O., \& Bülthoff, H. H. (2004). Merging the senses into a robust percept. Trends in Cognitive Sciences, 8, 162-169.

Exner, S. (1875). Experimentelle Untersuchung der einfachsten psychischen Processe [Experimental study of the most simple psychological processes]. Archiv für die Geschichte Physiologie (Pflüger's Arch.), 11, 403-432.

Farnè, A., \& Làdavas, E. (2002). Auditory peripersonal space in humans. Journal of Cognitive Neuroscience, 14, 1030-1043.

Fink, M., Ulbrich, P., Churan, J., \& Wittmann, M. (2006). Stimulusdependent processing of temporal order. Behavioural Processes, $71,344-352$.

Foxe, J. J., Wylie, G. R., Martinez, A., Schroeder, C. E., Javitt, D. C., Guilfoyle, D., et al. (2002). Auditory-somatosensory multisensory processing in auditory association cortex: An fMRI study. Journal of Neurophysiology, 88, 540-543.

Fraisse, P. (1980). Les synchronisations sensori-motrices aux rythmes [The sensorimotor synchronization of rhythms]. In J. Requin (Ed.), Anticipation et comportement [Sensorimotor synchronization to rhythms] (pp. 233-257). Paris: Centre National.

Francis, S. T., Kelly, E. F., Bowtell, R., Dunseath, W. J., Folger, S. E., \& McGlone, F. (2000). fMRI of the responses to vibratory stimulation of digit tips. NeuroImage, 11, 188-202.

Fu, K. G., Johnston, T. A., Shah, A. S., Arnold, L., Smiley, J., Hackett, T. A., et al. (2003). Auditory cortical neurons respond to somatosensory stimulation. Journal of Neuroscience, 23, 7510 7515.

Fucci, D., Petrosino, L., Harris, D., \& Randolph-Tyler, E. (1988). Auditory psychophysical scaling exposure effects: Magnitude estimation and cross-modal matching. Perceptual \& Motor Skills, 66, 643-648.

Fujisaki, W., \& Nishida, S. (2005). Temporal frequency characteristics of synchrony-asynchrony discrimination of audio-visual signals. Experimental Brain Research, 166, 455-464.

Fujisaki, W., \& Nishida, S. (2009). Audio-tactile superiority over visuo-tactile and audio-visual combinations in the temporal resolution of synchrony perception. Experimental Brain Research, $198,245-259$.

Fujisaki, W., \& Nishida, S. (2010). A common perceptual temporal limit of binding synchronous inputs across different sensory attributes and modalities. Proceedings of the Royal Society. Biological Sciences, 277, 2281-2290.

Fujisaki, W., Shimojo, S., Kashino, M., \& Nishida, S. (2004). Recalibration of audiovisual simultaneity. Nature Neuroscience, 7, 773-778.

Gardner, E. P., \& Spencer, W. A. (1972). Sensory funneling. II. Cortical neuronal representation of patterned cutaneous stimuli. Journal of Neurophysiology, 35, 954-977.

Geldard, F. A. (1985). The mutability of time and space on the skin. Journal of the Acoustical Society of America, 77, 233-237.

Geldard, F. A., \& Sherrick, C. E. (1972). The cutaneous "rabbit": A perceptual illusion. Science, 178, 178-179.

Gescheider, G. A. (1966). Resolving of successive clicks by the ears and skin. Journal of Experimental Psychology, 71, 378-381. 
Gescheider, G. A. (1967a). Auditory and cutaneous perceived successiveness. Journal of Experimental Psychology, 73, 179-186.

Gescheider, G. A. (1967b). Auditory and cutaneous temporal resolution of successive brief stimuli. Journal of Experimental Psychology, 75, 570-572.

Gescheider, G. A. (1970). Some comparisons between touch and hearing. IEEE Transactions on Man-Machine Systems, 11, 2835 .

Gibbon, J. (1977). Scalar expectancy theory and Weber's law in animal timing. Psychological Review, 84, 279-325.

Gilden, D. L., \& Marusich, L. R. (2009). Contraction of time in attention-deficit hyperactivity disorder. Neuropsychology, 23, 265-269.

Gillespie, P. G., \& Müller, U. (2009). Mechanotransduction by hair cells: Models, molecules, and mechanisms. Cell, 139, 33-44.

Glicksohn, J. (2001). Temporal cognition and the phenomenology of time: A multiplicative function for apparent duration. Consciousness and Cognition, 10, 1-25.

Goebl, W., \& Palmer, C. (2008). Tactile feedback and timing accuracy in piano performance. Experimental Brain Research, 186, 471479 .

Golaszewski, S. M., Siedentopf, C. M., Koppelstaetter, F., Fend, M., Ischebeck, A., Gonzalez-Felipe, V., et al. (2006). Human brain structures related to plantar vibrotactile stimulation: A functional magnetic resonance imaging study. NeuroImage, 29, 923929.

Gondan, M., Niederhaus, B., Rösler, F., \& Röder, B. (2005). Multisensory processing in the redundant-target effect: A behavioral and event-related potential study. Perception \& Psychophysics, 67, 713-726.

Goodfellow, L. D. (1934). An empirical comparison of audition, visual and touch in the discrimination of short intervals of time. American Journal of Psychology, 46, 243-258.

Gottlieb, G. (1971). Ontogenesis of sensory function in birds and mammals. In E. Tobach, L. R. Aronson, \& E. Shaw (Eds.), The biopsychology of development (pp. 67-128). New York: Academic.

Gregory, R. L. (1967). Origin of eyes and brains. Nature, 213, 369372.

Griffin, I. C., Miniussi, C., \& Nobre, A. C. (2001). Orienting attention in time. Frontiers in Bioscience, 6, 660-671.

Grondin, S. (2010). Timing and time perception: A review of recent behavioral and neuroscience findings and theoretical directions. Attention, Perception, \& Psychophysics, 72, 561-582.

Groome, L. J., Mooney, D. M., Holland, S. B., Smith, Y. D., Atterbury, J. L., \& Dykman, R. A. (2000). Temporal pattern and spectral complexity as stimulus parameters for eliciting a cardiac orienting reflex in human fetuses. Perception \& Psychophysics, 62, 313-320.

Grush, R. (2005). Internal models and the construction of time: Generalizing from state estimation to trajectory estimation to address temporal features of perception, including temporal illusions. Journal of Neural Engineering, 2, S209-S218.

Guest, S., Catmur, C., Lloyd, D., \& Spence, C. (2002). Audiotactile interactions in roughness perception. Experimental Brain Research, 146, 161-171.

Hackett, T. A., De La Mothe, L., Ulbert, I., Karmos, G., Smiley, J., \& Schroeder, C. E. (2007). Multisensory convergence in auditory cortex. II. Thalamocortical connections of the caudal superior temporal plane. Journal of Comparative Neurology, 502, 924952.

Hall, D. H., Edmondson-Jones, A. M., \& Fridriksson, J. (2006). Periodicity and frequency coding in human auditory cortex. European Journal of Neuroscience, 24, 3601-3610.

Hanson, J. V. M., Heron, J., \& Whitaker, D. (2008). Recalibration of perceived time across sensory modalities. Experimental Brain Research, 185, 347-352.
Hari, R. (1995). Illusory directional hearing in humans. Neuroscience Letters, 189, 29-30.

Harrar, V., \& Harris, L. R. (2008). The effect of exposure to asynchronous audio, visual, and tactile stimulus combinations on the perception of simultaneity. Experimental Brain Research, $186,517-524$.

Harris, J. A., Arabzadeh, E., Fairhall, A. L., Benito, C., \& Diamond, M. E. (2006). Factors affecting frequency discrimination of vibrotactile stimuli: Implications for cortical encoding. PLoS ONE, 1, 1-9.

Harris, D., Fucci, D., \& Petrosino, L. (1986). Lingual vibrotactile and auditory cross-modal matching: Frequency effects. Perceptual \& Motor Skills, 63, 1129-1130.

Harris, D., Fucci, D., \& Petrosino, L. (1989). Magnitude estimation and cross-modal matching of lingual vibrotactile and auditory sensation. Journal of Speech and Hearing Research, 32, 698702.

Hary, D., \& Moore, G. (1987). Synchronizing human movement with an external clock source. Biological Cybernetics, 56, 305-311.

Hatta, T., \& Ejiri, A. (1989). Learning effects of piano playing on tactile recognition of sequential stimuli. Neuropsychologia, 27, $1345-1356$.

Hernández, A., Salinas, E., García, R., \& Romo, R. (1997). Discrimination in the sense of flutter: New psychophysical measurements in monkeys. Journal of Neuroscience, 17, 63916400.

Hirsh, I. J., \& Fraisse, P. (1964). Simultanéité et succession de stimuli hétérogènes [Simultaneity and succession of heterogeneous stimuli]. Annales Médico-Psychologiques, 64, 1-19.

Hirsh, I. J., \& Sherrick, C. E., Jr. (1961). Perceived order in different sense modalities. Journal of Experimental Psychology, 62, 423432.

Hodinott-Hill, I., Thilo, K. V., Cowey, A., \& Walsh, V. (2002). Auditory chronostasis: Hanging on the telephone. Current Biology, 12, 1779-1781.

Hoh, J. K., Park, Y. S., Cha, K. J., \& Park, M. I. (2009). Fetal heart rate after vibroacoustic stimulation. International Journal of Gynaecology \& Obstetrics, 106, 14-18.

Holmes, N. P. (2007). The law of inverse effectiveness in neurons and behaviour: Multisensory integration versus normal variability. Neuropsychologia, 45, 3340-3345.

Holmes, N. P. (2009). The principle of inverse effectiveness in multisensory integration: Some statistical considerations. Brain Topography, 21, 168-176.

Holmes, N. P., \& Spence, C. (2005). Multisensory integration: Space, time and superadditivity. Current Biology, 15, R762-R764.

Hötting, K., Friedrich, C. K., \& Röder, B. (2009). Neural correlates of cross-modally induced changes in tactile awareness. Journal of Cognitive Neuroscience, 21, 2445-2461.

Hötting, K., \& Röder, B. (2004). Hearing cheats touch, but less in congenitally blind than in sighted individuals. Psychological Science, $15,60-64$.

Huang, J., Gamble, D., Wang, X., \& Hsiao, S. (2010). Auditory and tactile integration in music meter perception. Journal of the Acoustical Society of America, 127, 1949.

Iggo, A., \& Muir, A. R. (1969). The structure and function of a slowly adapting touch corpuscle in hairy skin. Journal of Physiology, 200, 763-796.

Iguchi, Y., Hoshi, Y., Nemoto, M., Taira, M., \& Hashimoto, I. (2007). Co-activation of the secondary somatosensory and auditory cortices facilitates frequency discrimination of vibrotactile stimuli. Neuroscience, 148, 461-472.

Ivry, R. B., \& Schlerf, J. E. (2008). Dedicated and intrinsic models of time perception. Trends in Cognitive Sciences, 12, 273-280.

Johansson, S., \& Vallbo, A. B. (1979a). Detection of tactile stimuli. Thresholds of afferent units related to psychophysical 
thresholds in the human hand. Journal of Physiology, 297, 405-422.

Johansson, S., \& Vallbo, A. B. (1979b). Tactile sensibility in the human hand: Relative and absolute densities of four types of mechanoreceptive units in glabrous skin. Journal of Physiology, 286, 283-300.

Jones, L. A., Poliakoff, E., \& Wells, J. (2009). Good vibrations: Human interval timing in the vibrotactile modality. Quarterly Journal of Experimental Psychology, 62, 71-86.

Jousmäki, V., \& Hari, R. (1998). Parchment-skin illusion: Soundbiased touch. Current Biology, 8, R190.

Katsuki, Y. (1965). Comparative neurophysiology of hearing. Physiological Reviews, 45, 380-423.

Kayser, C., \& Logothetis, N. K. (2007). Do early sensory cortices integrate cross-modal information? Brain Structure \& Function, $212,121-132$

Kayser, C., Petkov, C. I., Augath, M., \& Logothetis, N. K. (2005). Integration of touch and sound in auditory cortex. Neuron, 48, 373-378.

King, A. J. (2005). Multisensory integration: Strategies for synchronization. Current Biology, 15, R339-R341.

Kisilevsky, B. S. (1995). The influence of stimulus and subject variables on human fetal responses to sound and vibration. In J.P. Lecanuet, W. P. Fifer, N. A. Krasnegor, \& W. P. Smotherman (Eds.), Fetal development: A psychobiological perspective (pp. 263-278). Hillsdale: Erlbaum.

Kisilevsky, B. S., \& Muir, D. W. (1991). Human fetal and subsequent newborn responses to sound and vibration. Infant Behavior and Development, 14, 1-26.

Kisilevsky, B. S., Muir, D. W., \& Low, J. A. (1992). Maturation of human fetal responses to vibroacoustic stimulation. Child Development, 63, 1497-1508.

Kitagawa, N., \& Spence, C. (2006). Audiotactile multisensory interactions in information processing. Japanese Psychological Research, 48, 158-173.

Kitagawa, N., Zampini, M., \& Spence, C. (2005). Audiotactile interactions in near and far space. Experimental Brain Research, $166,528-537$.

Lagercrantz, H., \& Changeux, J. P. (2009). The emergence of human consciousness: From fetal to neonatal life. Pediatric Research, $65,255-260$.

LaMotte, R. H., \& Mountcastle, V. B. (1975). Capacities of humans and monkeys to discriminate vibratory stimuli of different frequency and amplitude: A correlation between neural events and psychological measurements. Journal of Neurophysiology, $38,539-559$.

Landis, C. (1954). Determinants of the critical flicker-fusion threshold. Physiological Reviews, 34, 259-286.

Lange, K., \& Röder, B. (2006). Orienting attention to points in time improves stimulus processing both within and across modalities. Journal of Cognitive Neuroscience, 18, 715-729.

Langers, D. R. M., Backes, W. H., \& van Dijk, P. (2007). Representation of lateralization and tonotopy in primary versus secondary human auditory cortex. NeuroImage, 34, 264-273.

Lappe, C., Herholz, S. C., Trainor, L. J., \& Pantev, C. (2008). Cortical plasticity induced by short-term unimodal and multimodal musical training. Journal of Neuroscience, 28, 9632-9639.

Large, E. W., \& Snyder, J. S. (2009). Pulse and meter as neural resonance. In Annals of the New York Academy of Sciences (Vol. 1169, pp. 46-57). Malden MS, USA:Wiley.

Lecaunet, J.-P., \& Schaal, B. (1996). Fetal sensory competencies. European Journal of Obstetrics \& Gynaecology and Reproductive Biology, 68, 1-23.

Lechelt, E. (1975). Temporal numerosity discrimination: Intermodal comparisons revisited. British Journal of Psychology, 66, 101-108.
Lee, J.-H., \& Spence, C. (2008). Assessing the benefits of multimodal feedback on dual-task performance under demanding conditions. In Proceedings of the 22nd British Computer Society HumanComputer Interaction Group Annual Conference (pp. 185-192). Liverpool, England: British Computer Society.

Lee, J.-H., \& Spence, C. (2009). Feeling what you hear: Taskirrelevant sounds modulates tactile perception delivered via a touch screen. Journal of Multisensory User Interfaces, 2, 145156.

Lestienne, R. (2001). Spike timing, synchronization and information processing on the sensory side of the central nervous system. Progress in Neurobiology, 65, 545-591.

Levänen, S., \& Hamdorf, D. (2001). Feeling vibrations: Enhanced tactile sensitivity in congenitally deaf humans. Neuroscience Letters, 301, 75-77.

Levitin, D. J., Maclean, K., Mathews, M., Chu, L., \& Jensen, E. (1999). The perception of cross-modal simultaneity (or "the Greenwich Observatory Problem" revisited). In D. M. Dubois (Ed.), Proceedings of Computing Anticipatory Systems: CASYS'99-Third International Conference, 9th-14th August (pp. 323-329). Liege, Belgium: Berlin, Heidelberg: Springer-Verlag

Lewkowicz, D. J. (2000). The development of intersensory temporal perception: An epigenetic systems/limitations view. Psychological Bulletin, 126, 281-308.

Ley, I., Haggard, P., \& Yarrow, K. (2009). Optimal integration of auditory and vibrotactile information for judgments of temporal order. Journal of Experimental Psychology: Human Perception and Performance, 35, 1005-1019.

Li Hegner, Y., Saur, R., Veit, R., Butts, R., Leiberg, S., Grodd, W., et al. (2007). BOLD adaptation in vibrotactile stimulation: Neuronal networks involved in frequency discrimination. Journal of Neurophysiology, 97, 264-271.

Lickliter, R. (2000). Atypical perinatal sensory stimulation and early perceptual development: Insights from developmental psychobiology. Journal of Perinatology, 20, S45-S54.

Lickliter, R., \& Bahrick, L. E. (2000). The development of infant intersensory perception: Advantages of a comparative convergent-operations approach. Psychological Bulletin, 126, $260-280$

Lim, V. K., Bradshaw, J. L., Nicholls, M. E., \& Altenmüller, E. (2005). Enhanced P1-N1 auditory evoked potential in patients with musicians' cramp. In Annals of the New York Academy of Sciences (Vol. 1060, pp. 349-359). Malden, MS, USA: Wiley.

Lloyd, D. M., Merat, N., McGlone, F., \& Spence, C. (2003). Crossmodal links between audition and touch in covert endogenous spatial attention. Perception \& Psychophysics, 65, 901-924.

Lotze, M., Scheler, G., Tan, H.-R. M., Braun, C., \& Birbaumer, N. (2003). The musician's brain: Functional imaging of amateurs and professionals during performance and imagery. NeuroImage, 20, 1817-1829.

Luna, R., Hernández, A., Brody, C. D., \& Romo, R. (2005). Neural codes for perceptual discrimination in primary somatosensory cortex. Nature Neuroscience, 8, 1210-1219.

Macefield, G., Gandevia, S. C., \& Burke, D. (1989). Conduction velocities of muscle and cutaneous afferents in the upper and lower limbs of human subjects. Brain, 112, 1519-1532.

Mahns, D. A., Perkins, N. M., Sahai, V., Robinson, L., \& Rowe, M. J. (2006). Vibrotactile frequency discrimination in human hairy skin. Journal of Neurophysiology, 95, 1442-1450.

Marks, L. E. (1983). Similarities and differences among the senses. International Journal of Neuroscience, 19, 1-11.

Mauk, M. D., \& Buonomano, D. V. (2004). The neural basis of temporal processing. Annual Review of Neuroscience, 27, 307-340.

Meck, W. H. (2005). Neuropsychology of timing and time perception. Brain and Cognition, 58, 1-8. 
Meck, W. H., \& Church, R. M. (1983). A mode control model of counting and timing processes. Journal of Experimental Psychology: Animal Behavior Processes, 9, 320-334.

Meredith, M. A., Nemitz, J. W., \& Stein, B. E. (1987). Determinants of multisensory integration in superior colliculus neurons. I. Temporal factors. Journal of Neuroscience, 7, 3215-3229.

Minagawa, H., Ohnishi, N., \& Sugie, N. (1996). Tactile-audio diagram for blind persons. IEEE Transactions on Rehabilitation Engineering, 4, 431-437.

Miniussi, C., Wilding, E. L., Coull, J. T., \& Nobre, A. C. (1999). Orienting attention in time. Modulation of brain potentials. Brain, 122, 1507-1518.

Mitterer, H., \& Jesse, A. (2010). Correlation versus causation in multisensory perception. Psychonomic Bulletin \& Review, 17, 329-334.

Moore, K. L., \& Persaud, T. V. N. (2008). The developing human: Clinically oriented embryology. Philadelphia: Saunders.

Morioka, M., \& Griffin, M. J. (2005). Thresholds for the perception of hand-transmitted vibration: Dependence on contact area and contact location. Somatosensory and Motor Research, 22, 281-297.

Morley, J. W., Vickery, R. M., Stuart, M., \& Turman, A. B. (2007). Suppression of vibrotactile discrimination by transcranial magnetic stimulation of primary somatosensory cortex. European Journal of Neuroscience, 26, 1007-1010.

Morokuma, S., Fukushima, K., Kawai, N., Tomonaga, M., Satoh, S., \& Nakano, H. (2004). Fetal habituation correlates with functional brain development. Behavioural Brain Research, $153,459-463$.

Mountcastle, V. B., Steinmetz, M. A., \& Romo, R. (1990). Frequency discrimination in the sense of flutter: Psychophysical measurements correlated with postcentral events in behaving monkeys. Journal of Neuroscience, 10, 3032-3044.

Müller, K., Aschersleben, G., Schmitz, F., Schnitzler, A., Freund, H. J., \& Prinz, W. (2008). Inter- versus intramodal integration in sensorimotor synchronization: A combined behavioral and magnetoencephalographic study. Experimental Brain Research, $185,309-318$

Murray, M. M., Molholm, S., Michel, C. M., Heslenfeld, D. J., Ritter, W., Javitt, D. C., et al. (2005). Grabbing your ear: Rapid auditory-somatosensory multisensory interactions in low-level sensory cortices are not constrained by stimulus alignment. Cerebral Cortex, 15, 963-974.

Nagarajan, S. S., Blake, D. T., Wright, B. A., Byl, N., \& Merzenich, M. M. (1998). Practice-related improvements in somatosensory interval discrimination are temporally specific but generalize across skin location, hemisphere, and modality. Journal of Neuroscience, 18, 1559-1570.

Navarra, J., Soto-Faraco, S., \& Spence, C. (2007). Adaptation to audiotactile asynchrony. Neuroscience Letters, 413, 72-76.

Nicolas, S. (1997). On the speed of different senses and nerve transmission by Hirsch (1862). Psychological Research, 59, 261-268.

Nicolson, T. (2005). Fishing for key players in mechanotransduction. Trends in Neurosciences, 28, 140-144.

Occelli, V., Spence, C., \& Zampini, M. (2008). Audiotactile temporal order judgments in blind and sighted individuals. Neuropsychologia, 46, 2845-2850.

Occelli, V., Spence, C., \& Zampini, M. (2009). The effect of sound intensity on the audiotactile crossmodal dynamic capture effect. Experimental Brain Research, 193, 409-419.

Oliveri, M., Koch, G., Salerno, S., Torriero, S., Lo Gerfo, E., \& Caltagirone, C. (2009). Representation of time intervals in the right posterior parietal cortex: Implications for a mental time line. NeuroImage, 46, 1173-1179.

Oliveri, M., Vicario, C. M., Salerno, S., Koch, G., Turriziani, P., Mangano, R., et al. (2008). Perceiving numbers alters time perception. Neuroscience Letters, 438, 308-311.
Oshurkova, E., Scheich, H., \& Brosch, M. (2008). Click train encoding in primary and non-primary auditory cortex of anesthetized macaque monkeys. Neuroscience, 153, 1289-1299.

Pantev, C., Lappe, C., Herholz, S. C., \& Trainor, L. (2009). Auditorysomatosensory integration and cortical plasticity in musical training. In Annals of the New York Academy of Sciences (Vol. 1169, pp.143-150). Malden MS, USA:Wiley.

Pantev, C., Ross, B., Fujioka, T., Trainor, L. J., Schulte, M., \& Schulz, M. (2003). Music and learning-induced cortical plasticity. In Annals of the New York Academy of Sciences (Vol. 999, pp. 438-450). Malden MS, USA:Wiley.

Patel, A. D., Iversen, J. R., Chen, Y., \& Repp, B. H. (2005). The influence of metricality and modality on synchronization with a beat. Experimental Brain Research, 163, 226-238.

Peck, J. E. (1994). Development of hearing. Part I: Phylogeny. Journal of the American Academy of Audiology, 5, 291-299.

Philippi, T. J., van Erp, J. B. F., \& Werkhoven, P. J. (2008). Multisensory temporal numerosity judgment. Brain Research, 1242, 116-125.

Phillips-Silver, J., \& Trainor, L. J. (2005). Feeling the beat: Movement influences infant rhythm perception. Science, 308, 1430.

Piéron, H. (1952). The sensations: Their functions, processes, and mechanisms. New Haven: Yale University Press.

Plack, C. J. (2004). Auditory perception. In M. W. Eysenck (Ed.), Psychology: An international perspective (pp. 1-20). London: Psychology Press.

Pollok, B., Müller, K., Aschersleben, G., Schnitzler, A., \& Prinz, W. (2004). The role of the primary somatosensory cortex in an auditorily paced finger tapping task. Experimental Brain Research, $156,111-117$.

Popescu, M., Otsuka, A., \& Ioannides, A. A. (2004). Dynamics of brain activity in motor and frontal cortical areas during music listening: A magnetoencephalographic study. NeuroImage, 21, $1622-1638$.

Pöppel, E. (1997). A hierarchical model of temporal perception. Trends in Cognitive Sciences, 1, 56-61.

Pöppel, E. (2009). Pre-semantically defined temporal windows for cognitive processing. Philosophical Transactions of the Royal Society of London. Series B, Biological Sciences, 364, 18871896.

Pöppel, E., Schill, K., \& von Steinbüchel, N. (1990). Sensory integration within temporally neutral system states: A hypothesis. Naturwissenschaften, 77, 89-91.

Popper, A. N. (2000). Hair cell heterogeneity and ultrasonic hearing: Recent advances in understanding fish hearing. Philosophical Transactions of the Royal Society of London. Series B, Biological Sciences, 355, 1277-1280.

Powers, A. R., III, Hillock, A. R., \& Wallace, M. T. (2009). Perceptual training narrows the temporal window of multisensory binding. Journal of Neuroscience, 29, 12265-12274.

Renier, L. A., Anurova, I., De Volder, A. G., Carlson, S., VanMeter, J., \& Rauschecker, J. P. (2009). Multisensory integration of sounds and vibrotactile stimuli in processing streams for "what" and "where". Journal of Neuroscience, 29, 10950-10960.

Repp, B. H. (2005). Multiple temporal references in sensorimotor synchronization with metrical auditory sequence. Psychological Research, 72, 79-98.

Repp, B. H., \& Penel, A. (2004). Rhythmic movement is attracted more strongly to auditory than to visual rhythms. Psychological Research, 68, 252-270.

Reyna, B. A., \& Pickler, R. H. (2009). Mother-infant synchrony. Journal of Obstetric, Gynaecologic, and Neonatal Nursing, 38 470-477.

Rivenez, M., Drake, C., Brochard, R., \& Guillaume, A. (2008). Selecting one of two regular sound sequences: Perceptual and motor effects of tempo. Perceptual \& Motor Skills, 106, 171-187. 
Ro, T., Hsu, J., Yasar, N. E., Caitlin Elmore, L., \& Beauchamp, M. S. (2009). Sound enhances touch perception. Experimental Brain Research, 195, 135-143.

Robles, L., \& Ruggero, M. A. (2001). Mechanics of the mammalian cochlea. Physiological Reviews, 81, 1305-1352.

Röder, B., \& Rösler, F. (2004). Compensatory plasticity as a consequence of sensory loss. In G. A. Calvert, C. Spence, \& B. E. Stein (Eds.), The handbook of multisensory processes (pp. 719-748). Cambridge: MIT Press.

Röder, B., Rösler, F., \& Spence, C. (2004). Early vision impairs tactile perception in the blind. Current Biology, 14, 121-124.

Röder, B., Teder-Saläjärvi, W., Sterr, A., Rösler, F., Hillyard, S. A., \& Neville, H. J. (1999). Improved auditory spatial tuning in blind humans. Nature, 400, 162-166.

Romani, G. L., Williamson, S. J., \& Kaufman, L. (1982). Tonotopic organization of the human auditory cortex. Science, 216, 13391340.

Romo, R., \& Salinas, E. (2003). Flutter discrimination: Neural codes, perception, memory and decision making. Nature Reviews Neuroscience, 4, 203-218.

Rowland, B. A., Quessy, S., Stanford, T. R., \& Stein, B. E. (2007). Multisensory integration shortens physiological response latencies. Journal of Neuroscience, 27, 5879-5884.

Rowland, B. A., \& Stein, B. E. (2008). Temporal profiles of response enhancement in multisensory integration. Frontiers in Neuroscience, 2, 218-224.

Rubinstein, T. C., Giladi, N., \& Hausdorff, J. M. (2002). The power of cueing to circumvent dopamine deficits: A review of physical therapy treatment of gait disturbances in Parkinson's disease. Movement Disorders, 17, 1148-1160.

Salinas, E., Hernandez, A., Zainos, A., \& Romo, R. (2000). Periodicity and firing rate as candidate neural codes for the frequency of vibrotactile stimuli. Journal of Neuroscience, 20 , $5503-5515$.

Sannita, W. G. (2000). Stimulus-specific oscillatory responses of the brain: A time/frequency-related coding process. Clinical Neurophysiology, 111, 565-583.

Schnupp, J. W. H., \& King, A. J. (2008). Auditory neuroscience: Neuronal sensitivity in humans. Current Biology, 18, R382-R385.

Schreiner, C. E., Read, H. L., \& Sutter, M. L. (2000). Modular organization of frequency integration in primary auditory cortex. Annual Review of Neuroscience, 23, 501-529.

Schroeder, C. E., \& Foxe, J. J. (2004). Multisensory convergence in early cortical processing. In G. A. Calvert, C. Spence, \& B. E. Stein (Eds.), The handbook of multisensory processes (pp. 295309). Cambridge: MIT Press.

Schroeder, C. E., \& Foxe, J. (2005). Multisensory contributions to lowlevel, 'unisensory' processing. Current Opinion in Neurobiology, $15,454-458$.

Schroeder, C. E., Lindsley, R. W., Specht, C., Marcovici, A., Smiley, J. F., \& Javitt, D. C. (2001). Somatosensory input to auditory association cortex in the macaque monkey. Journal of Neurophysiology, 85, 1322-1327.

Schulz, M., Ross, B., \& Pantev, C. (2003). Evidence for traininginduced crossmodal reorganization of cortical functions in trumpet players. NeuroReport, 14, 157-161.

Schürmann, M., Caetano, G., Hlushchuk, Y., Jousmäki, V., \& Hari, R. (2006). Touch activates human auditory cortex. NeuroImage, 30, $1325-1331$

Schutz, M., \& Kubovy, M. (2009). Causality and cross-modal integration. Journal of Experimental Psychology: Human Perception \& Performance, 35, 1791-1810.

Shams, L., Kamitani, Y., \& Shimojo, S. (2000). Illusions. What you see is what you hear. Nature, 408, 788.

Shams, L., Ma, W. J., \& Beierholm, U. (2005). Sound-induced flash illusion as an optimal percept. NeuroReport, 16, 1923-1927.
Sherrick, C. E., Jr. (1964). Effects of double simultaneous stimulation of the skin. American Journal of Psychology, 77, 42-53.

Siebert, W. M. (1970). Frequency discrimination in the auditory system: Place or periodicity mechanisms? Proceedings of the IEEE, 58, 723-730.

Smith, J. G., Harper, D. N., Gittings, D., \& Abernethy, D. (2007). The effect of Parkinson's disease on time estimation as a function of stimulus duration range and modality. Brain \& Cognition, 64, 130-143.

Soto-Faraco, S., \& Deco, G. (2009). Multisensory contributions to the perception of vibrotactile events. Behavioural Brain Research, 196, 145-154.

Spence, C. (in press). Multisensory perception, cognition, and behavior: Evaluating the factors modulating multisensory integration. To appear in B. E. Stein (Ed.), The new handbook of multisensory processing. Cambridge, MA: MIT Press.

Spence, C., Baddeley, R., Zampini, M., James, R., \& Shore, D. I. (2003). Multisensory temporal order judgments: When two locations are better than one. Perception \& Psychophysics, 65, 318-328.

Spence, C., \& Parise, C. (2010). Prior-entry: A review. Consciousness and Cognition, 19, 364-379.

Spence, C., Shore, D. I., \& Klein, R. M. (2001). Multisensory prior entry. Journal of Experimental Psychology: General, 130, 799-832.

Spence, C., \& Squire, S. (2003). Multisensory integration: Maintaining the perception of synchrony. Current Biology, 13, R519R521.

Stein, B. E., \& Meredith, M. A. (1993). The merging of the senses. Cambridge: MIT Press.

Stein, B. E., \& Stanford, T. R. (2008). Multisensory integration: Current issues from the perspective of the single neuron. Nature Reviews Neuroscience, 9, 255-267.

Sternberg, S., \& Knoll, R. L. (1973). The perception of temporal order: Fundamental issues and a general model. In S. Kornblum (Ed.), Attention \& performance, vol. 4 (pp. 629-685). London: Academic Press.

Sternberg, S., Knoll, R. L., \& Gates, B. A. (1971, November). Prior entry reexamined: Effect of attentional bias on order perception. Paper presented at the meeting of the Psychonomic Society, St. Louis, Missouri.

Stone, S. A. (1926). Prior entry in the auditory-tactual complication. American Journal of Psychology, 37, 284-287.

Talbot, W. H., Darian-Smith, I., Kornhuber, H. H., \& Mountcastle, V. B. (1968). The sense of flutter-vibration: Comparison of the human capacity with response patterns of mechanoreceptive afferents from the monkey hand. Journal of Neurophysiology, 31, 301-334.

Taubman, R. E. (1950a). Studies in judged number: I. The judgment of auditory number. Journal of General Psychology, 43, 195219.

Taubman, R. E. (1950b). Studies in judged number: II. The judgment of visual number. Journal of General Psychology, 43, 167-194.

Thaut, M. H. (2005). Rhythm, music and the brain: Scientific foundations and clinical applications. New York: Taylor \& Francis.

Titchener, E. B. (1908). Lectures on the elementary psychology of feeling and attention. New York: Macmillan.

Tramo, M. J., Cariani, P. A., Koh, C. K., Makris, N., \& Braida, L. D. (2005). Neurophysiology and neuroanatomy of pitch perception: Auditory cortex. In Annals of the New York Academy of Sciences (Vol. 1060, pp. 148-174). Malden MS, USA:Wiley.

Trehub, S. E., \& Hannon, E. E. (2006). Infant music perception: Domain-general or domain-specific mechanisms? Cognition, 100, 73-99.

Trojan, J., Getzmann, S., Möller, J., Kleinböhl, D., \& Hölzl, R. (2009). Tactile-auditory saltation: Spatiotemporal integration across sensory modalities. Neuroscience Letters, 460, 156-160.

Tsai, S. Y., Barnard, K. E., Lentz, M. J., \& Thomas, K. A. (2011). Mother-infant activity synchrony as a correlate of the emergence of circadian rhythm. Biological Research for Nursing, 13, 80-88. 
Ulrich, R., Nitschke, J., \& Rammsayer, T. (2006). Crossmodal temporal discrimination: Assessing the predictions of a general pacemaker-counter model. Perception \& Psychophysics, 68, $1140-1152$.

Van Damme, S., Gallace, A., Spence, C., Crombez, G., \& Moseley, G. L. (2009). Does the sight of physical threat induce a tactile processing bias? Modality specific facilitation of attention induced by viewing threatening pictures. Brain Research, 1253, 100-106.

van der Lubbe, R. H., Van Mierlo, C. M., \& Postma, A. (2010). The involvement of occipital cortex in the early blind in auditory and tactile duration discrimination tasks. Journal of Cognitive Neuroscience, 22, 1541-1556.

Van Eijk, R. L. J., Kohlrausch, A., Juola, J. F., \& van de Par, S. (2010). Temporal order judgment criteria are affected by synchrony judgment sensitivity. Attention, Perception, \& Psychophysics, 72, 2227-2235.

van Wassenhove, V. (2009). Minding time in an amodal representational space. Philosophical Transactions of the Royal Society of London. Series B, Biological Sciences, 364, 1815-1830.

van Wassenhove, V., Buonomano, D. V., Shimojo, S., \& Shams, L. (2008). Distortions of subjective time perception within and across senses. PLoS ONE, 3, e1437.

Vargas, J. G., \& Yu, W. (2008). Audio aided electro-tactile perception training for finger posture biofeedback. In Conference proceedings: Annual International Conference of the IEEE Engineering in Medicine and Biology Society. IEEE Engineering in Medicine and Biology Society (pp. 4230-4233). City, State: Publisher.

Vatakis, A., \& Spence, C. (2010). Audiovisual temporal integration for complex speech, object-action, animal call, and musical stimuli. In M. J. Naumer \& J. Kaiser (Eds.), Multisensory object perception in the primate brain (pp. 95-121). New York: Springer. Engineering in Medicine and Biology Society of the Institute of Electrical and Electronics Engineers.

Verrillo, R. T. (1985). Psychophysics of vibrotactile stimulation. Journal of the Acoustical Society of America, 77, 225-232.

Vicario, C. M., Pecoraro, P., Turriziani, P., Koch, G., \& Oliveri, M. (2008). Relativistic compression and extension of experiential time in the left and right space. PLoS ONE, 3, e1716.

Vicario, C. M., Rappo, G., Pepi, A. M., \& Oliveri, M. (2009). Timing flickers across sensory modalities. Perception, 38, 1144-1151.

Virsu, V., Oksanen-Hennah, H., Vedenpää, A., Jaatinen, P., \& LahtiNuuttila, P. (2008). Simultaneity learning in vision, audition, tactile sense and their cross-modal combinations. Experimental Brain Research, 186, 525-537.

von Baer, K. E. (1864). Welche Auffassung der lebenden Natur ist die richtige? [Which conception of nature is the correct one?]. In K. E. von Baer (Ed.), Reden gehalten in wissenschaftlichen Versammlungen und kleinere Aufsätze vermischten Inhalts. Theil 1: Reden (pp. 237-284). St. Petersburg: Schmitzdorff.

von Békésy, G. (1936). Low-frequency thresholds for hearing and feeling. Annalen der Physik, 26, 554-556.

von Békésy, G. (1955). Paradoxical direction of wave travel along the cochlear partition. Journal of the Acoustical Society of America, 27, 137-145.

von Békésy, G. (1957). Neural volleys and the similarity between some sensations produced by tones and skin vibrations. Journal of the Acoustical Society of America, 29, 1059-1069.

von Békésy, G. (1959). Similarities between hearing and skin sensations. Psychological Review, 66, 1-22.

von Békésy, G. (1963). Interaction of paired sensory stimuli and conduction in peripheral nerves. Journal of Applied Physiology, $18,1276-1284$.

von Békésy, G. (1967). Sensory inhibition. Princeton: Princeton University Press.
Vroomen, J., \& Keetels, M. (2010). Perception of intersensory synchrony: A tutorial review. Attention, Perception, \& Psychophysics, 72, 871-884.

Vroomen, J., Keetels, M., de Gelder, B., \& Bertelson, P. (2004). Recalibration of temporal order perception by exposure to audiovisual asynchrony. Cognitive Brain Research, 22, 32-35.

Vroomen, J., \& Stekelenburg, J. J. (2010). Visual anticipatory information modulates multisensory interactions of artificial audiovisual stimuli. Journal of Cognitive Neuroscience, 22, 1583-1596.

Wackermann, J. (2007). Inner and outer horizons of time experience. Spanish Journal of Psychology, 10, 20-32.

Wallace, M. T., Carriere, B. N., Perrault, T. J., Jr., Vaughan, J. W., \& Stein, B. E. (2006). The development of cortical multisensory integration. Journal of Neuroscience, 26, 11844-11849.

Walsh, V. (2003). A theory of magnitude: Common cortical metrics of time, space and quantity. Trends in Cognitive Sciences, 7, 483-488.

Wang, X., Lu, T., Bendor, D., \& Bartlett, E. (2008). Neural coding of temporal information in auditory thalamus and cortex. Neuroscience, 154, 294-303.

Watanabe, T. (1979). Funneling mechanism in hearing. Hearing Research, 1, 111-119.

Weiss, K., \& Scharlau, I. (2011). Simultaneity and temporal order perception: Different sides of the same coin? Evidence from a visual prior entry study. Quarterly Journal of Experimental Psychology, 64, 394-416.

Welch, R. B., \& Warren, D. H. (1980). Immediate perceptual response to intersensory discrepancy. Psychological Bulletin, 88, 638-667.

White, C. T., \& Cheatham, P. G. (1959). Temporal numerosity: IV. A comparison of the major senses. Journal of Experimental Psychology, 58, 441-444.

Wing, A. M., Doumas, M., \& Welchman, A. E. (2010). Combining multisensory temporal information for movement synchronisation. Experimental Brain Research, 200, 277-282.

Wittmann, M. (2009). The inner experience of time. Philosophical transactions of the Royal Society of London, Series B: Biological Sciences, 364, 1955-1967.

Woodward, S. C., \& Guidozzi, F. (1992). Intrauterine rhythm and blues? British Journal of Obstetrics \& Gynaecology, 99, 787-789.

Wozny, D. R., Beierholm, U. R., \& Shams, L. (2008). Human trimodal perception follows optimal statistical inference. Journal of Vision, 8, 1-11.

Xuan, B., Zhang, D., He, S., \& Chen, X. (2007). Larger stimuli are judged to last longer. Journal of Vision, 7, 1-5.

Yarrow, K., \& Rothwell, J. C. E. (2003). Manual chronostasis: Tactile perception precedes physical contact. Current Biology, 13, 1334 1339.

Yau, J. M., Olenczak, J. B., Dammann, J. F., \& Bensmaia, S. J. (2009). Temporal frequency channels are linked across audition and touch. Current Biology, 19, 561-566.

Zampini, M., Brown, T., Shore, D. I., Maravita, A., Röder, B., \& Spence, C. (2005). Audiotactile temporal order judgments. Acta Psychologica, 118, 277-291.

Zampini, M., Shore, D. I., \& Spence, C. (2003a). Multisensory temporal order judgments: The role of hemispheric redundancy. International Journal of Psychophysiology, 50, 165-180.

Zampini, M., Shore, D. I., \& Spence, C. (2003b). Audiovisual temporal order judgments. Experimental Brain Research, 152, 198-210.

Zatorre, R. J., Chen, J. L., \& Penhune, V. B. (2007). When the brain plays music: Auditory-motor interactions in music perception and production. Nature Reviews Neuroscience, 8, 547-558.

Zmigrod, S., Spapé, M., \& Hommel, B. (2009). Intermodal event files: Integrating features across vision, audition, taction, and action. Psychological Research, 73, 674-684. 